\title{
Application of the virtual fields method to the identification of the homogeneous anisotropic hardening parameters for advanced high strength steels
}

\author{
Jiawei Fu' , Frédéric Barlat ${ }^{\mathrm{a}}$, Jin-Hwan Kima,", Fabrice Pierron ${ }^{\mathrm{b}}$ \\ a Graduate Institute of Ferrous Technology, Pohang University of Science and Technology, \\ Pohang, Gyeongbuk 790-784, Republic of Korea, \\ jiaweifu@postech.ac.kr, f.barlat@postech.ac.kr, jinkim@postech.ac.kr \\ ${ }^{b}$ Faculty of Engineering and the Environment, University of Southampton, Southampton \\ SO17 1BJ, UK, F.Pierron@soton.ac.uk
}




\begin{abstract}
In the present paper, an inverse problem solution so called the virtual fields method (VFM) is implemented to identify the parameters of the homogeneous anisotropic hardening (HAH) model, a distortional plasticity-based model that describes the material plastic behavior when subjected to strain path changes. The framework of the identification method that combines the formulation of the yield condition, the constitutive stress-strain relation and the principle of virtual work is presented. For validation purpose, the proposed identification method was first attempted on finite element (FE) generated data for a forward-reverse simple shear test to investigate its capability in retrieving the input constitutive parameters. The influence of noise was also evaluated. Then, the identification method was applied to a selection of advanced high strength steel (AHSS), namely DP600, TRIP780 and TWIP980, sheet specimens, subjected to a small number of forward-reverse simple shear cycles. The material constitutive parameters were identified using the VFM based on which shear stress-strain curves were calculated and compared with their experimental counterparts. Good agreement was found between the calculated and the experimental curves despite the larger discrepancies observed in the reverse loading paths. To adjust these discrepancies, the original HAH model was modified with respect to the permanent softening related state variables. After modification, the model was simplified with only one state variable related to permanent softening. It was found that the discrepancies observed in the reverse loading paths were reduced with the modified HAH model.
\end{abstract}

Keywords: B. Constitutive behaviour; B. Metallic material; C. Finite elements; C. Mechanical testing; Constitutive parameters identification 


\section{Introduction}

Advanced high strength steels (AHSS) are widely used in the automotive industry due to their superior strength while maintaining high tensile elongation. Depending on the different requirements of automotive parts, e.g., crash worthiness, stiffness, lightweight, various types of AHSS can be chosen, such as dual-phase (DP), transformation-induced plasticity (TRIP) and twinning-induced plasticity (TWIP) steels. These steels exhibit various strength and maximum tensile elongation capabilities primarily due to different volume fractions of ferrite, martensite, bainite and austenite phases. During sheet metal forming, it is often challenging to achieve the desired shape with these materials since they generally exhibit lower ductility and larger elastic recovery, which, as a consequence, lead to the undesired springback phenomenon when forming loads are released (Firat et al., 2008; Haddag et al., 2007; Lee et al., 2005b; Yanagimoto et al., 2005).

As is well known, the Bauschinger effect and permanent softening play an important role in the springback phenomenon. The former corresponds to the decrease in reloading yield stress after a load reversal while the latter is related to the inability to recover the flow stress level of isotropic hardening. Efforts have been dedicated by many researchers to capturing these effects since isotropic hardening, which assumes that the yield surface expands proportionally, is not sufficient anymore for the prediction of springback, particularly using AHSS. Kinematic hardening models have been verified to be able to capture the Bauschinger effect and permanent softening as reviewed in (Chaboche, 2008). Various kinematic hardening models have been developed so far, from the early form that assumes that the yield surface translates in specific directions in the stress space while its shape is maintained (Prager, 1949; Ziegler, 1959), to the non-linear kinematic hardening models that combine both translation and expansion of the yield surface (Chaboche, 1986; Dafalias and Popov, 1975; Frederick and Armstrong, 2007; Ohno and Wang, 1993) and, more recently, the models that account for plastic anisotropy (Chung et al., 2005; Geng and Wagoner, 2002; Lee et al., 2005a; Lee et al., 2005b; Yoshida et al., 2015; Yoshida and Uemori, 2003). Alternatively, the distortional plasticity-based constitutive model denoted 'homogeneous anisotropic hardening (HAH)' (Barlat et al., 2011) can be used to capture these effects. Unlike the translation of the yield surface in kinematic hardening, the HAH model assumes that the yield surface both flattens on the opposite side of the active stress locus and isotropically expands (Manopulo et al., 2015). This model has advantages that it is expressed with a homogeneous 
function of degree one and reduces to isotropic hardening (with either an expanding isotropic or anisotropic yield surface) when the material is subjected to monotonic loading. This is a more realistic representation of material plastic behavior in practice. The finite element (FE) implementation of the HAH model has been successfully achieved by Lee et al. (2012), which is a useful tool to predict springback in metal forming. Very recently, the HAH model was extended to cross-loading cases with consideration of latent hardening effects (Barlat et al., 2013). It has also been refined to predict the mechanical response of metals subjected to non-proportional loading conditions (Barlat et al., 2014).

Increasing complexity in material models generally means that their experimental identification requires larger numbers of simple statically determinate tests. This is inconvenient and expensive, not even mentioning the issue related to obtaining the nominal target stress state because of inadequate boundary conditions and/or localization effects. With the strong development of strain mapping technology like digital image correlation (DIC) (Sutton et al., 2009), alternatives based on more complex test configurations have emerged. However, the static determination is generally lost in these, meaning that effective inverse tools are required to extract the constitutive parameters from the strain maps. Finite element model updating (FEMU) is a widely accepted inverse solution tool as reviewed in (Avril et al., 2008). It basically determines the material parameters by minimizing the discrepancy between FE simulation results and the experimental measurements. This has been applied over the years to a wide range of constitutive models, among which elasticity and elasto-plasticity (Broggiato et al., 2008; Güner et al., 2012; Lecompte et al., 2007; Wang et al., 2011; Zang et al., 2014; Zhang et al., 2014). Nevertheless, FEMU is computationally demanding since a FE model has to be updated iteratively, even for the simplest case of elasticity. An alternative is to use the virtual fields method (VFM) (Pierron and Grediac, 2012), an efficient inverse solution tool which is based on the principle of virtual work and takes full advantage of the availability of deformation maps through techniques such as DIC or the grid method (Grédiac et al., 2016), for instance. This approach determines the material parameters by minimizing the gap between the total internal and external virtual works (IVW and EVW) during the whole deformation process. The identification procedure is usually implemented using a simple MATLAB ${ }^{\circledR}$ program $^{1}$ and is very computationally efficient. So far, the VFM has been applied to elasticity, hyperelasticity, plasticity, and viscoplastictiy, for the characterization of

\footnotetext{
${ }^{1}$ A commercial software platform integrating DIC and VFM is now available, www.matchidmbc.com
} 
various materials (Fu et al., 2013; Grama et al., 2015; Grédiac and Pierron, 2006; Kim et al., 2012; Pierron et al., 2010). More work still needs to be done to extend the application of this method.

Currently, the identification of the HAH parameters is based on the FEMU approach in which the FE predicted stress-strain curve is updated iteratively to fit its experimental counterpart as done in (Choi et al., 2015). However, for the simple case of only one reverse loading path, this process generally takes a few days depending on the initial estimate as well as the deformation history. For more reverse loading cycles, the computation time is likely to be substantially longer. Therefore, it is highly desirable to develop a more efficient method to identify the HAH parameters.

In the present study, the VFM is extended to accommodate the HAH model. The proposed identification method is applied to the selected AHSS to identify their constitutive parameters when subjected to a few loading cycles. In Sections 2 and 3, the identification methodology is introduced step by step through an outline of the HAH formulation, the stress-strain constitutive relation and the principle of the VFM. In Section 4, the proposed identification method is validated on FE-generated data. The choice of virtual fields and the influence of measurement noise are also discussed. In Section 5, the proposed identification method is applied to experimental data from which the HAH parameters are identified for the selected AHSS. In Section 6, based on a discussion pertaining to the identification results, the original HAH model is modified to better describe the experimental response.

\section{Modeling of homogeneous anisotropic hardening}

\subsection{Framework of the HAH model}

As proposed by Barlat et al. (2011), the HAH yield function is comprised of two components, namely $\varphi$ and $\varphi_{h}$. The former is a stable component, which describes the original yield surface shape. Any isotropic or anisotropic yield function can be used as the stable component, depending on the material anisotropy. The latter is a fluctuating component which describes the distortion of the yield surface during loading. The corresponding yield condition is expressed as:

$$
\Phi(\boldsymbol{s})=\left[\varphi^{q}+\varphi_{h}^{q}\right]^{\frac{1}{q}}=\left[\varphi^{q}(\boldsymbol{s})+f_{1}^{q}|\widehat{\boldsymbol{h}}: \boldsymbol{s}-| \widehat{\boldsymbol{h}}: \boldsymbol{s}||^{q}+f_{2}^{q}|\widehat{\boldsymbol{h}}: \boldsymbol{s}+| \widehat{\boldsymbol{h}}: \boldsymbol{s}||^{q}\right]^{\frac{1}{q}}=\sigma(\bar{\varepsilon})
$$


where $\boldsymbol{s}$ is the stress deviator and $\widehat{\boldsymbol{h}}$ the microstructure deviator. The distortion of the yield surface is controlled by the evolution of the state variables $f_{1}$ and $f_{2}$ as well as the double dot product $\widehat{\boldsymbol{h}}: \boldsymbol{s}$ through the operation shown in Eq. (1). It is worth noting that $\varphi$ is selected as a homogeneous yield function of first degree with respect to the stress deviator. Due to the constant exponent $q$ (normally chosen as 2), the yield function $\Phi(\boldsymbol{s})$ is also homogeneous of first degree and is equal to the current reference flow stress $\sigma(\bar{\varepsilon})$ under the work-equivalent effective plastic strain $\bar{\varepsilon}$. Another advantage of this yield function is that, in the case of monotonic loading, the yield surface keeps the same shape as that of isotropic hardening on the side of the active stress while flattening distortion occurs on the opposite side. This provides a more accurate prediction of material plastic behavior over kinematic hardening models.

As introduced earlier, the fluctuating component $\varphi_{h}$ controls the distortion of the yield surface. In this component, the normalized tensorial state variable $\widehat{\boldsymbol{h}}$ describes the material microstructure evolution during deformation and determines the rotation direction of the yield surface such as in the case of cross-loading. The initial value of $\widehat{\boldsymbol{h}}$ is given by

$$
\widehat{\boldsymbol{h}}_{0}=\frac{\boldsymbol{s}_{0}}{\sqrt{\frac{8}{3} s_{0}: s_{0}}}
$$

where $\boldsymbol{s}_{0}$ is the stress deviator that leads to the first plastic strain increment. $f_{1}$ and $f_{2}$, on the other hand, determine the amount of distortion so that the HAH model can capture the Bauschinger effect and permanent softening after a load reversal. These two state variables are substituted by another set of two state variables $g_{1}$ and $g_{2}$, which are easier to interpret physically, through the relation

$$
f_{k}=\left[\frac{1}{g_{k}^{q}}-1\right]^{\frac{1}{q}}
$$

where $k$ takes the values of 1 and 2. One can see that the current yield stress is determined by $g_{l}$ and $g_{2}$, which are functions of two additional state variables $g_{3}$ and $g_{4}$. The initial values for $g_{l} \sim g_{4}$ are

$$
g_{1}^{0}=g_{2}^{0}=g_{3}^{0}=g_{4}^{0}=1, \text { when } \bar{\varepsilon}=0
$$

All these state variables $g_{l} \sim g_{4}$ and $\widehat{\boldsymbol{h}}$ evolve with an increase of the effective plastic strain $\bar{\varepsilon}$. The laws for their evolutions depend on the sign of $\widehat{\boldsymbol{h}}: \boldsymbol{s}$. 
For $\widehat{\boldsymbol{h}}: \boldsymbol{s} \geq 0$

$$
\begin{aligned}
\frac{d g_{1}}{d \bar{\varepsilon}} & =k_{2}\left(k_{3} \frac{\sigma(0)}{\sigma(\bar{\varepsilon})}-g_{1}\right) \\
\frac{d g_{2}}{d \bar{\varepsilon}} & =k_{1} \frac{g_{3}-g_{2}}{g_{2}} \\
\frac{d g_{4}}{d \bar{\varepsilon}} & =k_{5}\left(k_{4}-g_{4}\right) \\
\frac{d \widehat{\boldsymbol{h}}}{d \bar{\varepsilon}} & =k\left[\hat{\boldsymbol{s}}-\frac{8}{3} \widehat{\boldsymbol{h}}(\hat{\boldsymbol{s}}: \widehat{\boldsymbol{h}})\right]
\end{aligned}
$$

For $\widehat{\boldsymbol{h}}: \boldsymbol{s}<0$

$$
\begin{aligned}
\frac{d g_{1}}{d \bar{\varepsilon}} & =k_{1} \frac{g_{4}-g_{1}}{g_{1}} \\
\frac{d g_{2}}{d \bar{\varepsilon}} & =k_{2}\left(k_{3} \frac{\sigma(0)}{\sigma(\bar{\varepsilon})}-g_{2}\right) \\
\frac{d g_{3}}{d \bar{\varepsilon}} & =k_{5}\left(k_{4}-g_{3}\right) \\
\frac{d \widehat{\boldsymbol{h}}}{d \bar{\varepsilon}} & =k\left[-\widehat{\boldsymbol{s}}+\frac{8}{3} \widehat{\boldsymbol{h}}(\hat{\boldsymbol{s}}: \widehat{\boldsymbol{h}})\right]
\end{aligned}
$$

where $\hat{\boldsymbol{s}}$ represents the normalized stress deviator calculated as in Eq. (2), $\hat{\boldsymbol{s}}=\frac{\boldsymbol{s}}{\sqrt{\frac{8}{3} s: s}} \cdot k_{1} \sim k_{5}$ and $k$ are material constitutive parameters, among which $k_{1}, k_{2}, k_{3}$ are associated to the Bauschinger effect, $k_{4}, k_{5}$ are associated to permanent softening, and $k$ is associated to the rotation of the yield surface, i.e., when loading path changes to cross-loading for instance.

Simultaneously with distortion, the yield surface expands at a rate given by the hardening law for monotonic loading in the HAH model, i.e., Swift hardening law in the present work. Therefore,

$$
\sigma(\bar{\varepsilon})=C\left(\varepsilon_{0}+\bar{\varepsilon}\right)^{n}
$$

where $C, \varepsilon_{0}$ and $n$ are Swift hardening parameters.

In order to use the HAH model to predict material deformation behavior when subjected to cyclic loadings using, e.g., in FE simulations, the above material parameters must be determined a priori. In the present study, only reverse loading is considered. Thus, the parameter $k$ is empirically chosen as a known value, here 30 to keep consistent with previous studies, because, according to the previous study (Barlat et al., 2011), this parameter does not influence the material behavior in reverse loading. The eight parameters (von Mises for $\varphi$ ) $k_{1} \sim k_{5}, C, \varepsilon_{0}$ and $n$, however, are unknown and need to be identified from experiments. 


\subsection{Stress determination}

In order to identify the material parameters, it is necessary to know the constitutive stress-strain relation so that the stress values during elasto-plastic deformation can be determined from the strain values since, in practice, stress is not directly measurable. This is an essential step for the VFM inverse problem solution, which will be explained later in Section 3. Here, a stress calculation algorithm that was developed by Sutton et al. (1996) and was later applied by Kim et al. (2013) is adopted. The constitutive equation of elasticity is

$$
d \boldsymbol{\sigma}=\boldsymbol{Q}: d \boldsymbol{\varepsilon}_{e}
$$

where $d \boldsymbol{\sigma}, d \boldsymbol{\varepsilon}_{e}$ are the stress and elastic strain increment vector, respectively and $\boldsymbol{Q}$ the stiffness matrix. Since the total strain increment $d \boldsymbol{\varepsilon}$ is composed of the elastic part $d \boldsymbol{\varepsilon}_{e}$ and the plastic part $d \boldsymbol{\varepsilon}_{p}$, thus

$$
d \boldsymbol{\sigma}=\boldsymbol{Q}:\left(d \boldsymbol{\varepsilon}-d \boldsymbol{\varepsilon}_{p}\right)
$$

For the yield condition

$$
f=\Phi(\sigma)-\sigma(\bar{\varepsilon})=0
$$

with the associated flow rule

$$
d \boldsymbol{\varepsilon}_{p}=d \lambda \frac{\partial f}{\partial \boldsymbol{\sigma}}
$$

where $d \lambda(\geq 0)$ is the plastic multiplier. As the stresses should remain on the yield surface, $d f$ thus equals zero. In this case,

$$
d f=\frac{\partial f}{\partial \sigma}: d \sigma+\frac{\partial f}{\partial \bar{\varepsilon}} d \bar{\varepsilon}=0
$$

Combining Eqs. (9)-(12), one can finally obtain the elasto-plastic stress-strain relation which writes:

$$
d \boldsymbol{\sigma}=\left(\boldsymbol{Q}-\frac{\boldsymbol{Q}: \frac{\partial f}{\partial \boldsymbol{\sigma}} \otimes \boldsymbol{Q}: \frac{\partial f}{\partial \boldsymbol{\sigma}}}{\frac{\partial f}{\partial \boldsymbol{\sigma}}: \boldsymbol{Q}: \frac{\partial f}{\partial \boldsymbol{\sigma}}-\frac{\partial f}{\partial \bar{\varepsilon}}}\right): d \boldsymbol{\varepsilon}
$$

Based on Eq. (13), the stress evolution can be determined from the total strain which, in practice, can be measured using various deformation measurement techniques. 


\section{Inverse problem solution using the VFM}

At this stage, the objective is to solve the inverse problem so that the HAH parameters can be determined. The VFM is applied to solve this. The VFM is based on the principle of virtual work. For a continuous deformable solid with volume $V$ subjected to a traction vector $\boldsymbol{T}$ on the boundary $\partial V$, if the body force is negligible and the loading is quasi-static, an integral form of the equilibrium equation can be written:

$$
-\int_{V} \boldsymbol{\sigma}: \boldsymbol{\varepsilon}^{*} d V+\int_{\partial V} \boldsymbol{T} \cdot \boldsymbol{u}^{*} d S=0
$$

where $\boldsymbol{u}^{*}$ is the selected virtual displacement vector, and $\boldsymbol{\varepsilon}^{*}$ is the virtual strain tensor derived from $\boldsymbol{u}^{*}$. A virtual displacement field is essentially a test function that verifies the equilibrium of Eq. (14). This equilibrium equation is true for any virtual field $\boldsymbol{u}^{*}$ as long as it is continuous and differentiable. However, in practice, it is advantageous to define $\boldsymbol{u}^{*}$ as zero over the fixed boundary so that the virtual work done by the unknown reaction force can be cancelled out from the equilibrium equation, and to define $\boldsymbol{u}^{*}$ as constant over the moving boundary which allows the equilibrium equation to contain the measured resulting load. During elasto-plastic deformation, the stress tensor $\boldsymbol{\sigma}$ at time $t$ in this equation can be calculated as:

$$
\boldsymbol{\sigma}=\int_{0}^{t} \dot{\boldsymbol{\sigma}} d t
$$

where $\dot{\boldsymbol{\sigma}}=\frac{d \boldsymbol{\sigma}}{d t}$ is the stress rate tensor. Thus, Eq. (14) becomes

$$
-\int_{V} \int_{0}^{t} \dot{\boldsymbol{\sigma}} d t: \boldsymbol{\varepsilon}^{*} d V+\int_{\partial V} \boldsymbol{T} \cdot \boldsymbol{u}^{*} d S=0
$$

Based on the above equilibrium equation, a cost function that measures the gap between the internal and external virtual work (IVW and EVW), which correspond to the first and the second term in Eq. (16), respectively, can be formed

$$
\mathcal{g}(\boldsymbol{X})=\sum_{i=1}^{N_{i}}\left(-\int_{V}\left(\int_{0}^{t} \dot{\boldsymbol{\sigma}} d t\right): \boldsymbol{\varepsilon}^{*} d V+\int_{\partial V} \boldsymbol{T} \cdot \boldsymbol{u}^{*} d S\right)^{2}
$$

where $N_{i}$ is the total number of loading increments. In this cost function, the traction $\boldsymbol{T}$ can be measured, the virtual fields $\boldsymbol{u}^{*}$ and $\boldsymbol{\varepsilon}^{*}$ are pre-selected, and the stress increments $d \boldsymbol{\sigma}$ can be substituted by the unknown material parameters $\boldsymbol{X}$ and the strain increments $d \boldsymbol{\varepsilon}$ obtained from fullfield deformation measurements through the relation in Eq. (13). Therefore, $g(\boldsymbol{X})$ is a function of 
the unknown constitutive parameters. By minimizing $\mathcal{g}(\boldsymbol{X})$ with respect to $\boldsymbol{X}$, the unknown parameters can determined.

For the present work, all the tested sheet specimens are assumed to be in plane stress as the thickness $b$ is in the order of $1 \mathrm{~mm}$, thus

$$
g(\boldsymbol{X})=\sum_{i=1}^{N_{i}}\left(-b \int_{A}\left(\int_{0}^{t} \dot{\boldsymbol{\sigma}} d t\right): \boldsymbol{\varepsilon}^{*} d S+\int_{\partial V} \boldsymbol{T} \cdot \boldsymbol{u}^{*} d S\right)^{2}
$$

where $A$ is the area of interest (AOI). Note that, in the present study, the selected $\boldsymbol{u}^{*}$ should be spatially constant over the traction boundary so that the EVW of the measured resulting force $\boldsymbol{F}$ can be easily calculated.

The above proposed identification methodology has been implemented using MATLAB ${ }^{\circledR}$. A minimization algorithm based on nonlinear least-squares, "Isqnonlin" available in MATLAB ${ }^{\circledR}$, is adopted to minimize the quadratic gap between the IVW and the EVW in the cost function $g(\boldsymbol{X})$. Note that an initial estimate for the unknown parameters must be provided to start the minimization algorithm.

\section{Numerical validation}

Before applying the proposed VFM scheme to real experimental data, it is necessary to examine its adaptability and efficiency in identifying the constitutive parameters from full-field deformation data. To verify this, a FE model of forward-reverse simple shear was developed usingABAQUS ${ }^{\circledR}$. The simulated strain and force data were exported from ABAQUS ${ }^{\circledR}$ and input into the VFM program to obtain the constitutive parameters. The identified values were compared to the reference input values. By doing this, the accuracy of the identification procedure can be evaluated. Then, random noise was added to the simulated data. The idea is to evaluate the influence of measurement noise on the identification accuracy.

\subsection{FE analysis and identification results}

The schematic of the FE model, which simulates the current experimental configuration, is illustrated in Fig. 1. As can be seen in Fig. 1a, the left boundary of the rectangular specimen is fixed and the right boundary translates with the moving grip. The dimensions of the specimen are $50 \times 4 \mathrm{~mm}$, for $l_{1} \times l_{2}$, respectively. A 2D shell model is used with a thickness of $1 \mathrm{~mm}$. It should be mentioned that several mesh sizes $(0.3 \mathrm{~mm}, 0.5 \mathrm{~mm}$ and $0.7 \mathrm{~mm})$ have been tested for the FE 
model. Since no apparent difference was observed among the identification results, only the results corresponding to $0.5 \mathrm{~mm}$ are shown in this paper. The input material parameters, which are given in Table 1, were selected based on the HAH distortional model with Swift isotropic hardening. Note that, for the sake of simplicity, von Mises yield criterion was chosen for the stable component $\varphi$ in Eq. (1). However, in the case of cross-loading, depending on the material anisotropy, more suitable yield criteria such as YLD2000-2D (Barlat et al., 2003) should be used. As illustrated in Fig. 1b, the specimen was deformed in a loading mode of forward-reverse simple shear. Here, one forward loading path together with the subsequent reverse one (i.e., LP1+LP2) are regarded as one loading cycle. The specimen was deformed with two and a half cycles. In each loading path, 0.5 $\mathrm{mm}$ displacement was applied to the moving grip. Each loading path was divided into 100 loading increments. The logarithmic strain at each element centroid and the applied force at each node of the right boundary were exported from ABAQUS ${ }^{\circledR}$ at each loading increment for identification purpose. In order to investigate the effect of loading path on the identification results, the constitutive parameters were identified based on different loading cycles.

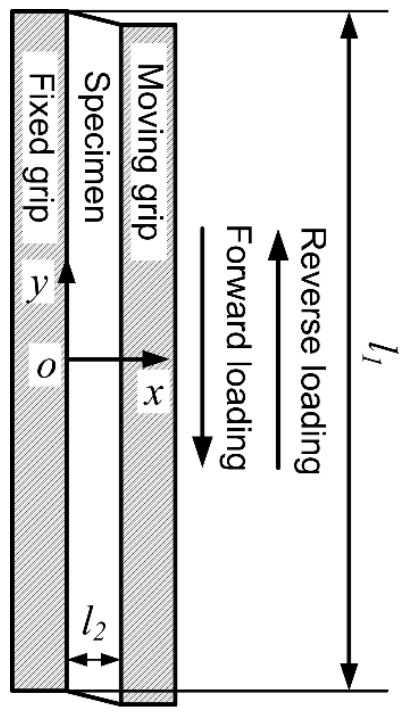

(a)

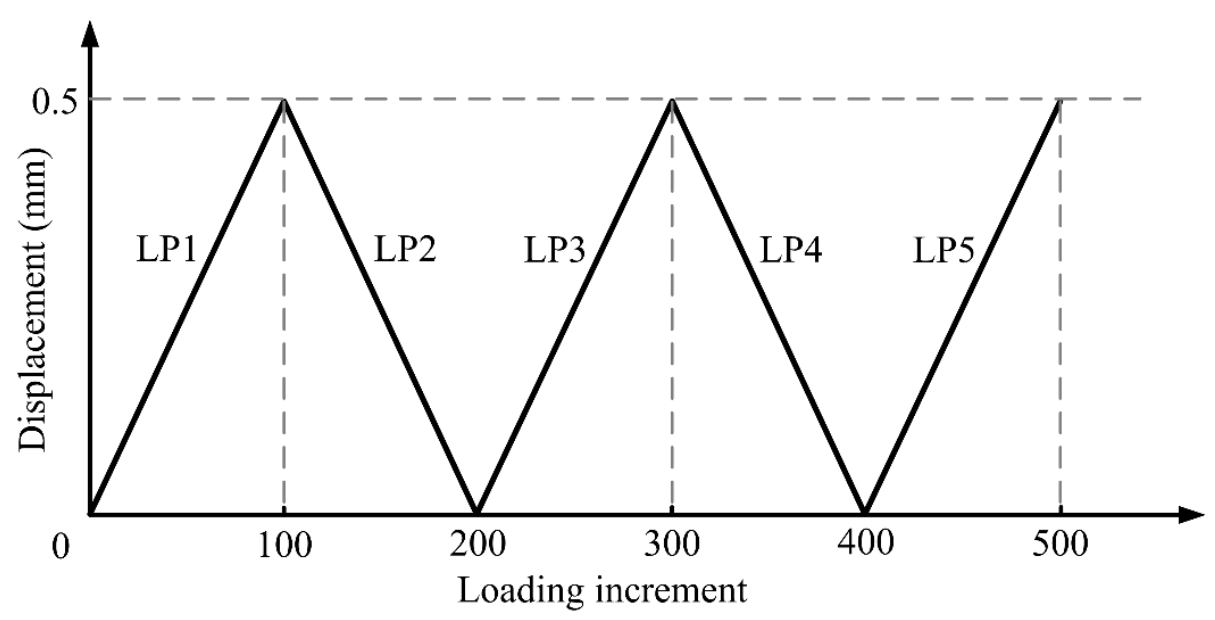

(b)

Fig. 1. FE model of the forward-reverse simple shear test, (a) schematic of the test configuration and (b) loading mode 
Table 1 Reference constitutive parameters for the FE model

\begin{tabular}{cccccccccccc}
\hline$E(\mathrm{GPa})$ & $v$ & $k_{1}$ & $k_{2}$ & $k_{3}$ & $k_{4}$ & $k_{5}$ & $k$ & $q$ & $C(\mathrm{MPa})$ & $\varepsilon_{0}$ & $n$ \\
\hline 200 & 0.3 & 150 & 20 & 0.5 & 0.5 & 5 & 30 & 2 & 1000 & 0.005 & 0.25 \\
\hline
\end{tabular}

As explained earlier, to facilitate the calculation of the EVW, the selected virtual fields should be zero over the fixed boundary and constant over the moving boundary. First, the simplest virtual field was selected, denoted VF1, which can be written as:

$$
V F 1: u_{x}^{*}=0, u_{y}^{*}=x \Rightarrow \varepsilon_{x x}^{*}=0, \varepsilon_{y y}^{*}=0, \varepsilon_{x y}^{*}=0.5
$$

where $x$ is the horizontal coordinate of each element centroid.

Based on VF1, the constitutive parameters, namely $k_{1} \sim k_{5}, C, \varepsilon_{0}$ and $n$, were identified from different numbers of loading cycles. For each case, two different initial estimates were used to start the minimization process. This is to check the robustness of the algorithm in providing a global solution. The identified parameters are listed in Table 2 and their relative errors to the reference values are plotted in Fig. 2. From these results, one can observe that for the case of two loading paths (LP1-LP2, only one loading cycle), the HAH parameters are not correctly retrieved, especially for $k_{2} \sim k_{5}$. The results are highly dependent on the choice of initial estimate, indicating that one loading cycle cannot provide a unique solution for the HAH parameters. When the loading cycles are increased to one and a half (LP1-LP3), the identification results become stable and unique for different initial estimates, although the discrepancy for $k_{4}$ and $k_{5}$ is relatively large. Further improvement can be observed when the loading cycles are increased to two (LP1-LP4). In this case, all the identified HAH and Swift hardening constitutive parameters are in good agreement with their reference counterparts. Also, the solutions are identical for the two initial estimates. When further increasing the loading cycles to two and a half (LP1-LP5), no significant difference is observed. The global identification errors are very close to the case of LP1-LP4. Thus, it can be concluded from these results that two forward-reverse loading cycles are compulsory for an accurate identification of the HAH parameters. Regarding computation time, for the cases of LP1-LP2 and LP1-LP3, the computation time is highly dependent on the choice of the initial estimates, ranging from half an hour to several hours. However, for LP1-LP4 and LP1-LP5, the computation time is no longer than half an hour for all cases (with 32-bit operating system, 3.20 $\mathrm{GHz}$ CPU, and $8.00 \mathrm{~GB}$ RAM). This is much faster than the conventional FEMU identification 
method (Choi et al., 2015) which has been used to identify the HAH parameters and, normally, takes a few days. Thus, the LP1-LP4 loading history was chosen for the experimental applications for the sake of accurate identification at minimum cost.

Table 2 Identification results obtained with the simulated data using VF1

\begin{tabular}{ccccccccc}
\hline & $k_{1}$ & $k_{2}$ & $k_{3}$ & $k_{4}$ & $k_{5}$ & $C(M P a)$ & $\varepsilon_{0}$ & $n$ \\
\hline Reference values & 150 & 20 & 0.5 & 0.5 & 5 & 1000 & 0.005 & 0.25 \\
\hline Initial estimate 1 & 100 & 50 & 0.1 & 0.1 & 1 & 800 & 0.01 & 0.1 \\
\hline LP1-LP2 & 159 & 12.7 & 0.144 & 0.0846 & 2.64 & 1020 & 0.00528 & 0.256 \\
LP1-LP3 & 154 & 21.1 & 0.524 & 0.565 & 6.26 & 1025 & 0.00545 & 0.258 \\
LP1-LP4 & 155 & 19.5 & 0.483 & 0.511 & 5.36 & 1017 & 0.00526 & 0.255 \\
LP1-LP5 & 152 & 19.5 & 0.491 & 0.510 & 5.35 & 1020 & 0.00538 & 0.256 \\
\hline Initial estimate 2 & 200 & 10 & 0.8 & 0.8 & 10 & 1200 & 0.001 & 0.3 \\
\hline LP1-LP2 & 158 & 19.5 & 0.467 & 0.846 & 84.5 & 1021 & 0.00529 & 0.256 \\
LP1-LP3 & 154 & 21.1 & 0.524 & 0.567 & 6.31 & 1026 & 000546 & 0.258 \\
LP1-LP4 & 155 & 19.5 & 0.483 & 0.511 & 5.36 & 1017 & 0.00526 & 0.255 \\
LP1-LP5 & 152 & 19.5 & 0.491 & 0.510 & 5.35 & 1020 & 0.00538 & 0.256 \\
\hline
\end{tabular}




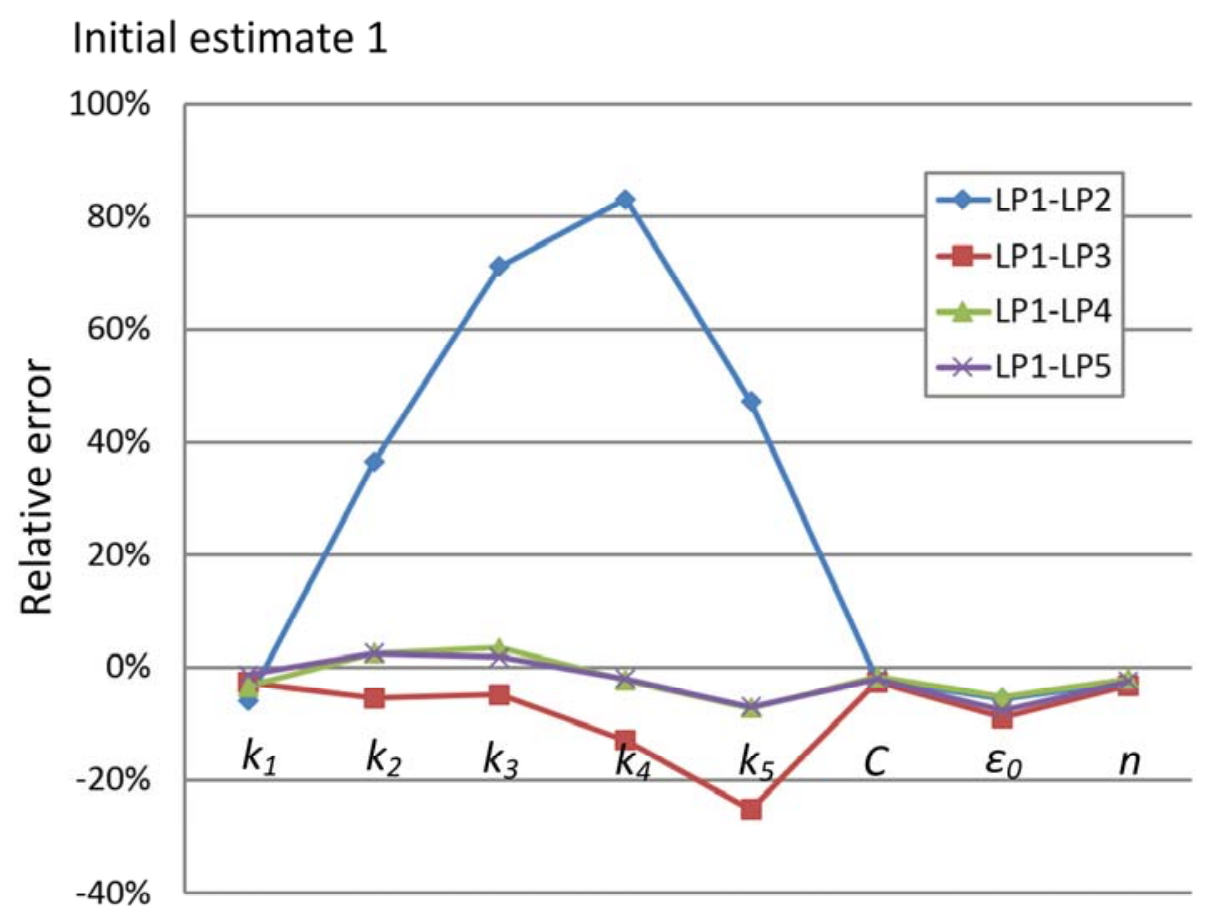

Initial estimate 2

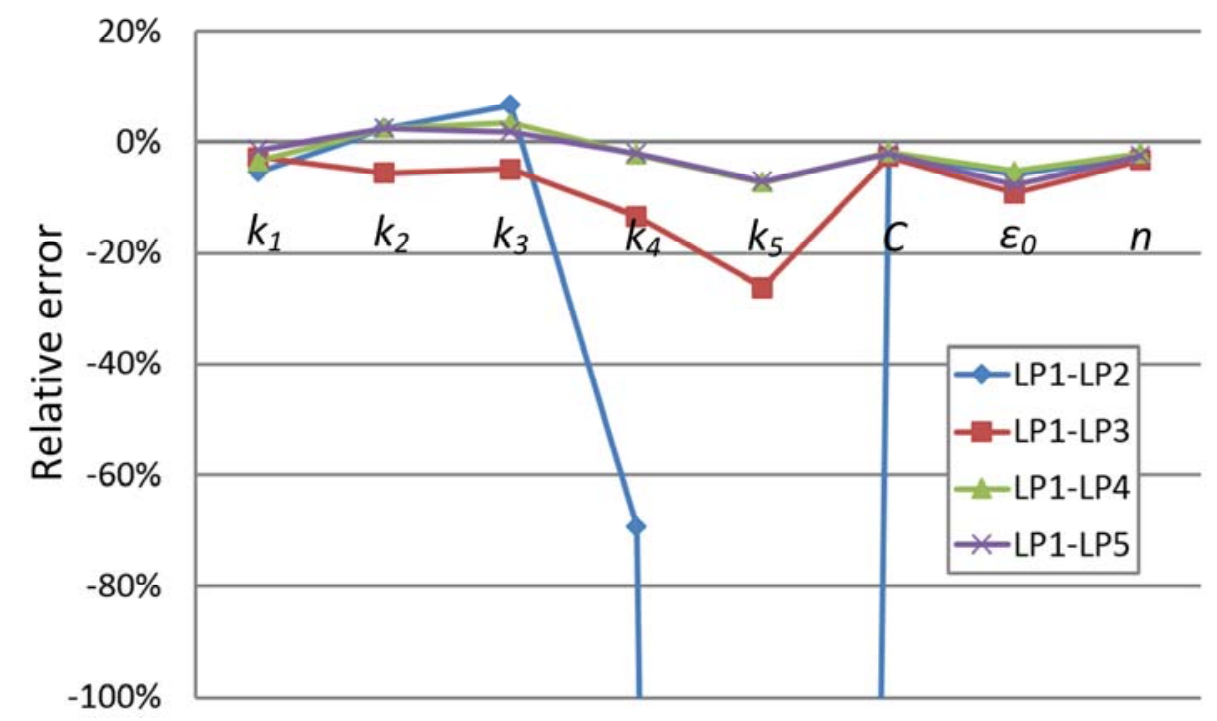

Fig. 2. Relative errors of identified constitutive parameters for both initial estimates.

In order to evaluate the reliability of the identification results, the shear stress-strain curves calculated by the reference parameters and the identified counterparts are compared in Fig. 3, plotted in black solid line and red circles, respectively. Here, the parameters obtained with the Initial estimate 1 and loading mode LP1-LP4 were used. It is clear that the curve calculated by the identified parameters is in very good agreement with the reference curve, confirming that the identification results are reliable. 


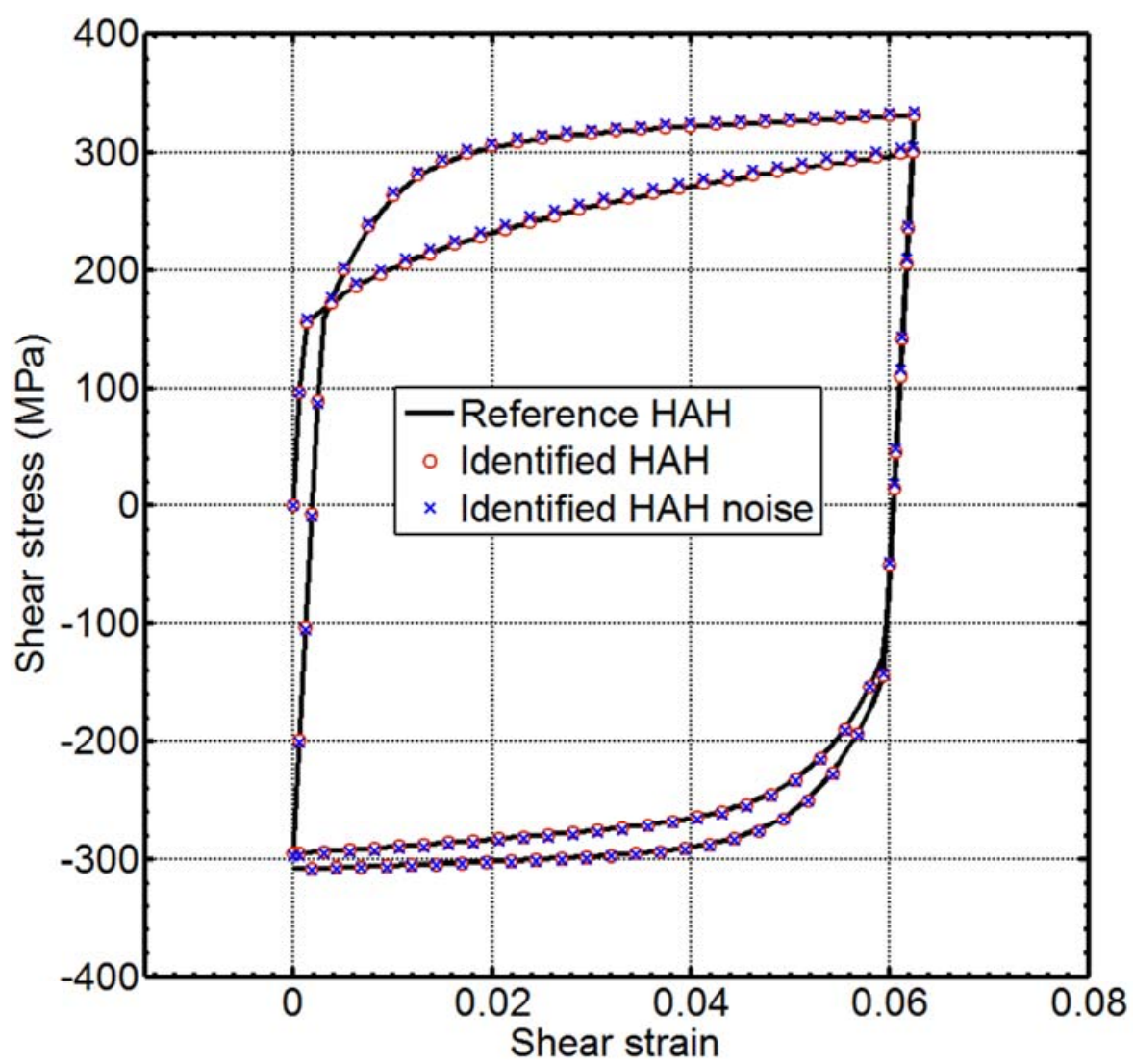

Fig. 3. Shear stress-strain curves calculated by the identified parameters with and without noise (using the Identified 1 and VF1 in Table 2 and Table 4, loading mode LP1-LP4) v.s. the reference curve.

It is interesting to investigate the influence of the choice of virtual fields on the identification results. Thus, two other sets of virtual fields have been tried, which are

$$
\begin{gathered}
V F 2: u_{x}^{*}=\sin x \sin y \sin \left(x-l_{2}\right), u_{y}^{*}=x \\
V F 3: u_{x}^{*}=\sin y \sin \left(\frac{\pi x}{l_{2}}\right), u_{y}^{*}=x^{2}
\end{gathered}
$$

Compared to the uniform virtual shear strain in VF1, the above virtual fields provide heterogeneous virtual shear strains which depend on $x$ through the operations of sine and cosine functions. The identification results obtained with the initial estimate 1 and loading mode LP1LP4 for the above three selected virtual fields are listed in Table 3. It can be observed that for the different virtual fields, the identification results are very close to each other, indicating that the choice of virtual fields does not significantly influence the identification of the HAH and Swift 
hardening parameters in this case. Therefore, for the sake of simplicity, only VF1 is considered in the rest of this paper.

Table 3 Identification results for the three selected virtual fields

\begin{tabular}{ccccccccc}
\hline & $k_{1}$ & $k_{2}$ & $k_{3}$ & $k_{4}$ & $k_{5}$ & $C(\mathrm{MPa})$ & $\varepsilon_{0}$ & $n$ \\
\hline Reference values & 150 & 20 & 0.5 & 0.5 & 5 & 1000 & 0.005 & 0.25 \\
\hline Initial estimate 1 & 100 & 50 & 0.1 & 0.1 & 1 & 800 & 0.01 & 0.1 \\
\hline VF1 & 155 & 19.5 & 0.483 & 0.511 & 5.36 & 1017 & 0.00526 & 0.255 \\
VF2 & 155 & 19.5 & 0.480 & 0.511 & 5.38 & 1019 & 0.00530 & 0.256 \\
VF3 & 155 & 19.5 & 0.485 & 0.511 & 5.34 & 1016 & 0.00524 & 0.255 \\
\hline
\end{tabular}

\subsection{Influence of noise}

Since the strain measurement is polluted by different sources of noise in practice, the identification results can be affected. Therefore, another objective of this preliminary study is to evaluate the influence of noise. In the present work, digital image correlation (DIC) was utilized for strain measurement. More details regarding this will be given in Section 5. In order to determine the noise level of strain measurement, a series of stationary speckle images of an undeformed specimen were taken successively and logarithmic strains were calculated using DIC. Then, the strain resolutions for all strain components were obtained by calculating their standard deviations, which are $1.92 \times 10^{-4}, 1.72 \times 10^{-4}$, and $1.42 \times 10^{-4}$ for $\varepsilon_{x x}, \varepsilon_{y y}$, and $\varepsilon_{x y}$, respectively. The mean values of all the strain components are very close to zero, $\sim 10^{-5}$. It should be mentioned that this is a favourable situation. In practice, displacements are noisy and spatial smoothing is required to reduce strain noise, which may create systematic errors in the strain field. A more realistic way to evaluate the performance would be to use speckle image deformation as in (Wang et al., 2016). In this work, to simulate the above noise, a random Gaussian white noise with the corresponding amplitudes obtained from the experiments was added to the simulated strain data for the whole deformation process (LP1-LP4). The noise-polluted strain data were then used to feed the proposed VFM scheme to obtain the constitutive parameters. The results are listed in Table 4. One can see that for the three selected virtual fields, the identification results are very close to each other. As expected, the identification error is increased after adding the noise, particularly for $k_{3}$ and $k_{5}$. The optimized virtual fields as introduced in (Pierron et al., 2010) may reduce this bias, which, 
however, is beyond the scope of the present paper. In Fig. 3, the shear stress-strain curve calculated by the identified parameters (VF1) using the noise-polluted data is compared to the curve without noise as well as the reference one. It is clear that despite the slight deviation (mostly in LP1, caused by the errors in the identified Swift hardening parameters), the noise polluted curve is still in good agreement with the reference curve. This indicates that the noise in the experiments does not significantly affect the robustness of the proposed VFM identification scheme in characterizing the reverse loading behavior.

Table 4 Identification results for the noise-polluted strain data (using VF1-3, loading mode LP1LP4)

\begin{tabular}{ccccccccc}
\hline & $k_{1}$ & $k_{2}$ & $k_{3}$ & $k_{4}$ & $k_{5}$ & $C(M P a)$ & $\varepsilon_{0}$ & $n$ \\
\hline Reference values & 150 & 20 & 0.5 & 0.5 & 5 & 1000 & 0.005 & 0.25 \\
\hline Initial estimate 1 & 100 & 50 & 0.1 & 0.1 & 1 & 800 & 0.01 & 0.1 \\
\hline VF1 & 155 & 19.1 & 0.446 & 0.513 & 5.65 & 1027 & 0.00531 & 0.253 \\
VF2 & 155 & 19.3 & 0.448 & 0.512 & 5.68 & 1029 & 0.00538 & 0.254 \\
VF3 & 155 & 19.1 & 0.446 & 0.514 & 5.66 & 1026 & 0.00527 & 0.252 \\
\hline
\end{tabular}

\section{Applications on AHSS}

\subsection{Materials and experiments}

At this stage, the proposed identification method is ready to be applied to real experimental data. Three types of AHSS, namely DP600, TRIP780 and TWIP980 were investigated. The specimens were cut from bigger sheets with $1.42 \mathrm{~mm}, 1.43 \mathrm{~mm}$ and $1.25 \mathrm{~mm}$ thickness for the DP600, TRIP780 and TWIP980, respectively.

The specimens were installed in the simple shear test device which was driven by a $500 \mathrm{kN}$ MTS $^{\circledR}$ universal tension-compression machine. A detailed description of the test device can be found in (Choi et al., 2015). Briefly, the specimens were tested exactly as illustrated in Fig. 1(a). The shear deformation direction was along the metal rolling direction. An advantage of the present forward-reverse simple shear test configuration is that it was specially designed to avoid buckling of the specimen so that it can reach a high strain level in reverse loading. This is usually a limitation 
for tension-compression tests for sheet metal specimens as buckling arises at an early stage of compression. The tests were performed based on a mode of displacement control with a low crosshead speed of $0.01 \mathrm{~mm} . \mathrm{s}^{-1}$ for all specimens, corresponding to a strain rate of $1.25 \times 10^{-3} \mathrm{~s}^{-1}$. The strain level at each loading path is about $5 \%$. This value varies for the three selected steels due to the compliance of the test device induced by the different material strengths. Nevertheless, this is not a critical issue as it does not affect the effectiveness of the proposed identification method. Two cycles of forward-reverse simple shear were conducted for each specimen according to the loading mode LP1-LP4 as suggested in the FE simulation. Two specimens were tested for each material to check the repeatability of the results.

A stereo digital image correlation (SDIC) system was employed to measure the full-field deformations during the tests. Before measurement, a random speckle pattern was sprayed on the specimen surface. For each specimen, a speckle image was taken as the reference state before deformation and subsequently one image per second was recorded during deformation. At the meantime, synchronized load data were recorded by the load cell of the MTS machine. The image size is $2048 \times 2448$ pixels along the directions of $x$ and $y$, respectively. It should be mentioned that only the central region $(20 \times 4 \mathrm{~mm}$ along the directions of $y$ and $x$, respectively) of the specimen is within the field of view for the sake of a good spatial resolution along the $x$-direction. One such speckle image for the DP600 specimen 1 is illustrated in Fig. 4. Indeed, if the whole specimen (50 $\times 4 \mathrm{~mm}$ ) is within the field of view, there will be very limited number of pixels along the direction $x$ for DIC calculation. For DIC, the AOI is divided into smaller subsets. The displacement vector of each subset is determined by tracking and matching the speckles of the subset in the reference and deformed states. This was implemented using the commercial DIC software VIC-3D. The information on DIC, the hardware and the post-processing parameters are listed in Table 5. Fig. 5 illustrates a speckle image of the subsets with $33 \times 33$ pixels subset size and the magnified view of one subset. 
Table 5 Imaging and measurement performance information (DP600 specimen 1)

\begin{tabular}{|c|c|}
\hline Technique used & Stereo Digital Image Correlation \\
\hline Sensor and digitization & $2048 \times 2448,14$-bit \\
\hline Lens, camera angle and imaging distance & $75 \mathrm{~mm} \mathrm{C}$-mount, $17.2^{\circ}, 0.33 \mathrm{~m}$ \\
\hline Total number of images & $573(1 \mathrm{~Hz})$ \\
\hline Pixel to $\mathrm{mm}$ conversion & 1 pixel $=0.011 \mathrm{~mm}$ \\
\hline AOI (pixels or mm) & $20 \mathrm{~mm} \times 4 \mathrm{~mm}(1830 \times 360$ pixels $)$ \\
\hline Subset (SS), step (ST) & 33,11 \\
\hline $\begin{array}{l}\text { Interpolation, shape functions, correlation } \\
\text { criterion }\end{array}$ & $\begin{array}{l}\text { Cubic spline, affine, Normalized squared } \\
\text { differences }\end{array}$ \\
\hline Pre-smoothing & N/A \\
\hline Displacement Resolution & $\begin{array}{l}\mathrm{u}_{\mathrm{x}}=1.67 \times 10^{-4} \mathrm{~mm}(0.015 \text { pixels }) \\
\mathrm{u}_{\mathrm{y}}=2.76 \times 10^{-4} \mathrm{~mm}(0.025 \text { pixels }) \\
\mathrm{u}_{\mathrm{z}}=1.0 \times 10^{-3} \mathrm{~mm}(0.09 \text { pixels })\end{array}$ \\
\hline \multicolumn{2}{|l|}{ Strain } \\
\hline Smoothing technique & Local polynomial - affine \\
\hline Strain window $(\mathrm{SW})$ & 9 data points \\
\hline $\begin{array}{l}\text { Virtual strain gauge size } \\
\mathrm{VSG}=[(\mathrm{SW}-1) \times \mathrm{ST}]+\mathrm{SS}\end{array}$ & 121 pixels, $1.33 \mathrm{~mm}$ \\
\hline Resolution & $\begin{array}{l}\varepsilon_{x x}=1.92 \times 10^{-4} \\
\varepsilon_{y y}=1.72 \times 10^{-4} \\
\varepsilon_{x y}=1.42 \times 10^{-4}\end{array}$ \\
\hline
\end{tabular}

It should be mentioned that due to the discrete nature of the deformation measurement, the integrals in the cost function Eq. (18) must be approximated by discrete sums. In this case, $\boldsymbol{g}(\boldsymbol{X})$ becomes

$$
g(\boldsymbol{X}) \approx \sum_{i=1}^{N_{i}}\left(-b \sum_{j=1}^{N_{e}}\left(\sum_{0}^{t} d \boldsymbol{\sigma}\right): \boldsymbol{\varepsilon}^{*} S^{(j)}+\boldsymbol{F} \cdot \boldsymbol{u}^{*}\right)^{2}
$$

where $N_{e}$ stands for the total number of the discrete elements in deformation measurements over the AOI, and $S^{(j)}$ is the area of the $j^{\text {th }}$ element. This is an approximation which quality depends on the spatial resolution of the measurements. The only way to evaluate the related error is to simulate 
the complete identification chain using synthetically deformed speckle images as proposed in (Rossi et al., 2015).

Fig. 4 also shows the measured load data versus time and the logarithmic shear strain maps exported from DIC at different time steps for the DP600 specimen 1 in two cycles of forwardreverse simple shear test. In these maps, strain concentrations can be observed on both sides of the specimen. This is not surprising as the grips have a serrated groove that was specially designed to prevent the specimen from slipping during the test. It should be mentioned that the logarithmic strain values that were used to identify the material constitutive parameters in the VFM program were derived through the analytical procedure and the finite deformation theory as explained in (Avril et al., 2010; Kim et al., 2012). Briefly, the AOI on the specimen is meshed with triangular elements (0.5 mm mesh size) as shown in Fig. 6 using a Delaunay triangularization algorithm. In the undeformed state, since the coordinates of each element node and the scattered measurement points inside the element are predefined known values, a basis of piecewise linear functions that fit the scattered data points onto the nodal points thus can be determined using least squares regression. Using these piecewise functions and the deformed coordinates of scattered measurement points, the nodal coordinates in the deformed states can be determined. Then, based on the nodal coordinates in the underformed and deformed states, the deformation gradient and the logarithmic strain tensor can be calculated using the finite deformation theory. An advantage of this method is that it allows the measurement of nodal coordinates near edges, which are not obtainable in the DIC technique. The shear stress-strain curve for the DP600 specimen 1 is illustrated in Fig. 7. In this figure, the shear stress $\sigma_{x y}$ is calculated by the ratio between the measured load and the cross sectional area along the direction $y$ while the shear strain $\varepsilon_{x y}$ is given by the mean shear strain value in the AOI measured with DIC. Shear modulus $G$ is calculated from the slope of the shear stress-strain curve in the initial linear range. Young's modulus $E$ can be deduced from $G$ using the relation

$$
E=2 G(1+v)
$$

where Poisson's ratio $v$ is set to 0.3 , which is a reasonable approximation for the selected AHSS according to (Chung et al., 2011; Kim et al., 2013). The calculated Young's moduli are 212, 209 and $192 \mathrm{GPa}$ for the DP600, TRIP780 and TWIP980 specimens, respectively, which are reasonable values. 

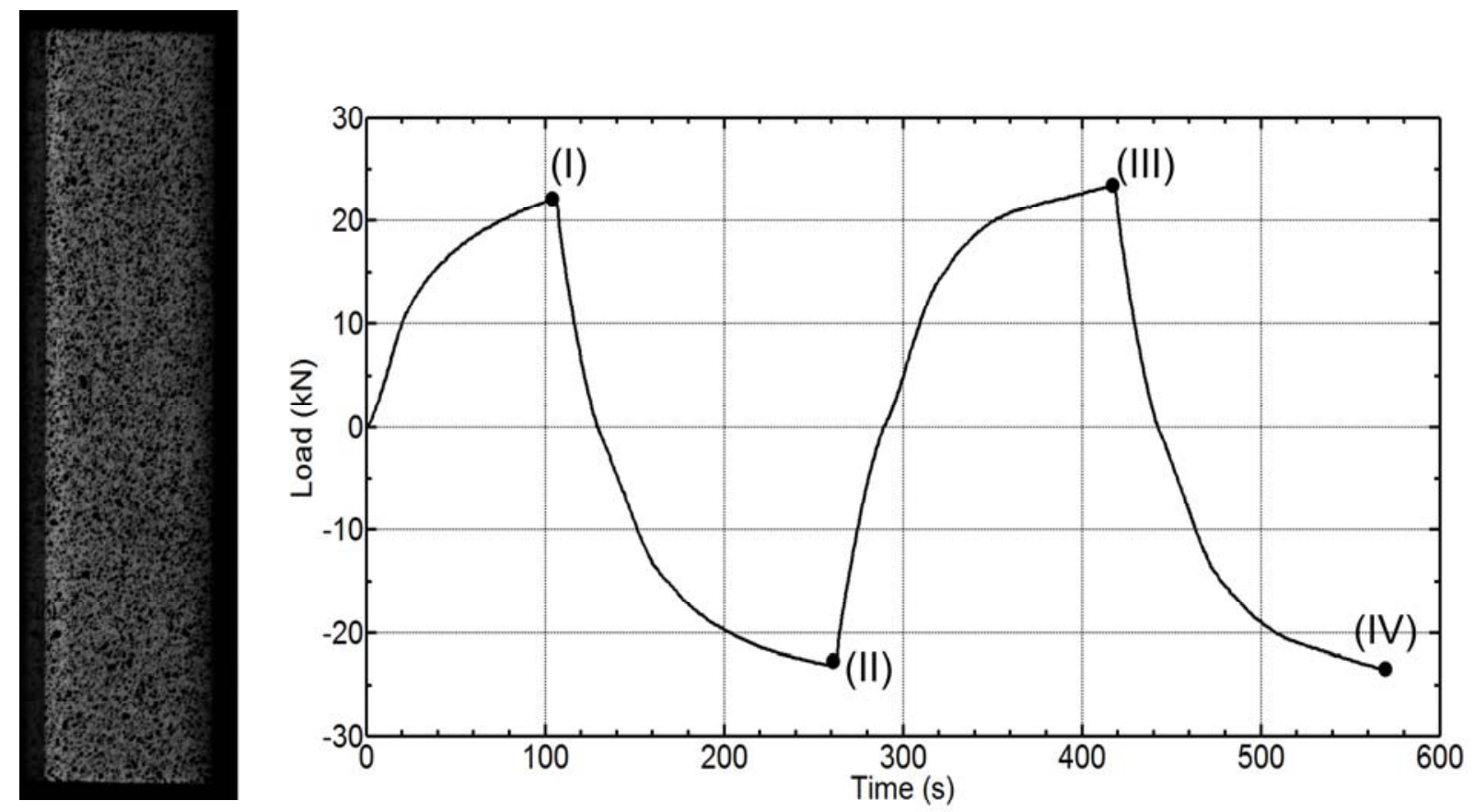

\section{$\varepsilon_{x y}$}

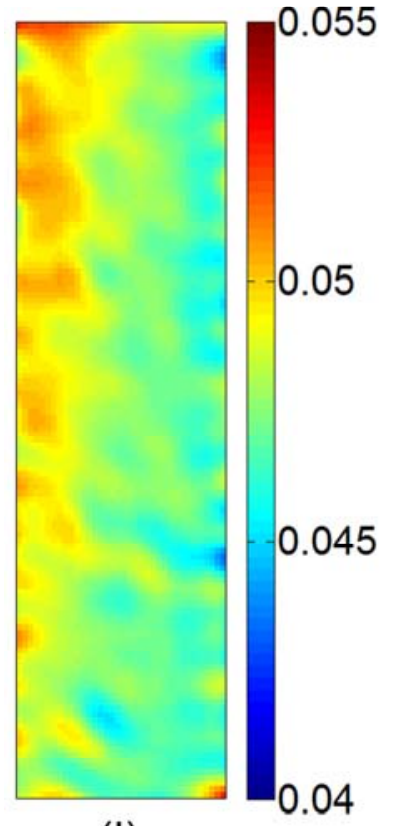

(I)

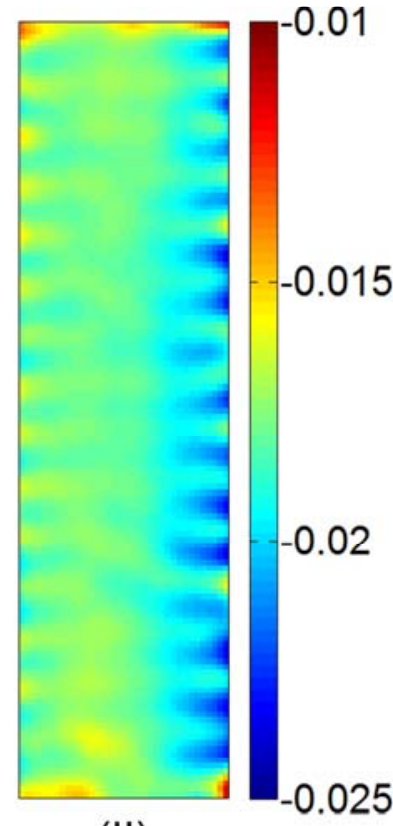

(II)

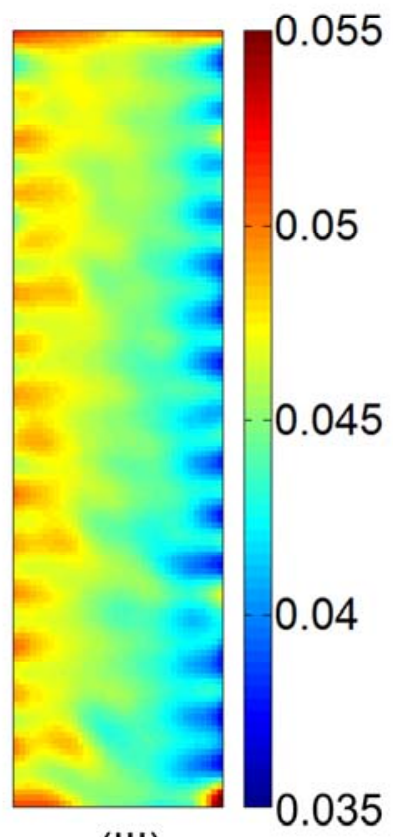

(III)

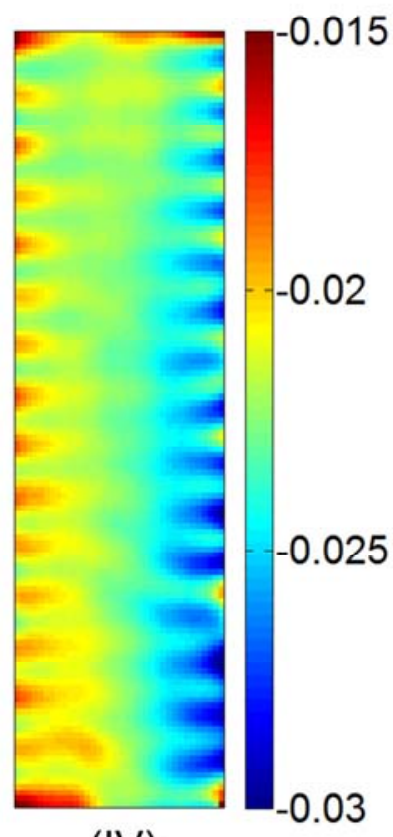

(IV)

Fig. 4 A speckle image, the load curve and the logarithmic shear strain maps at different time steps for the DP600 specimen 1 in two cycles of forward-reverse simple shear test 


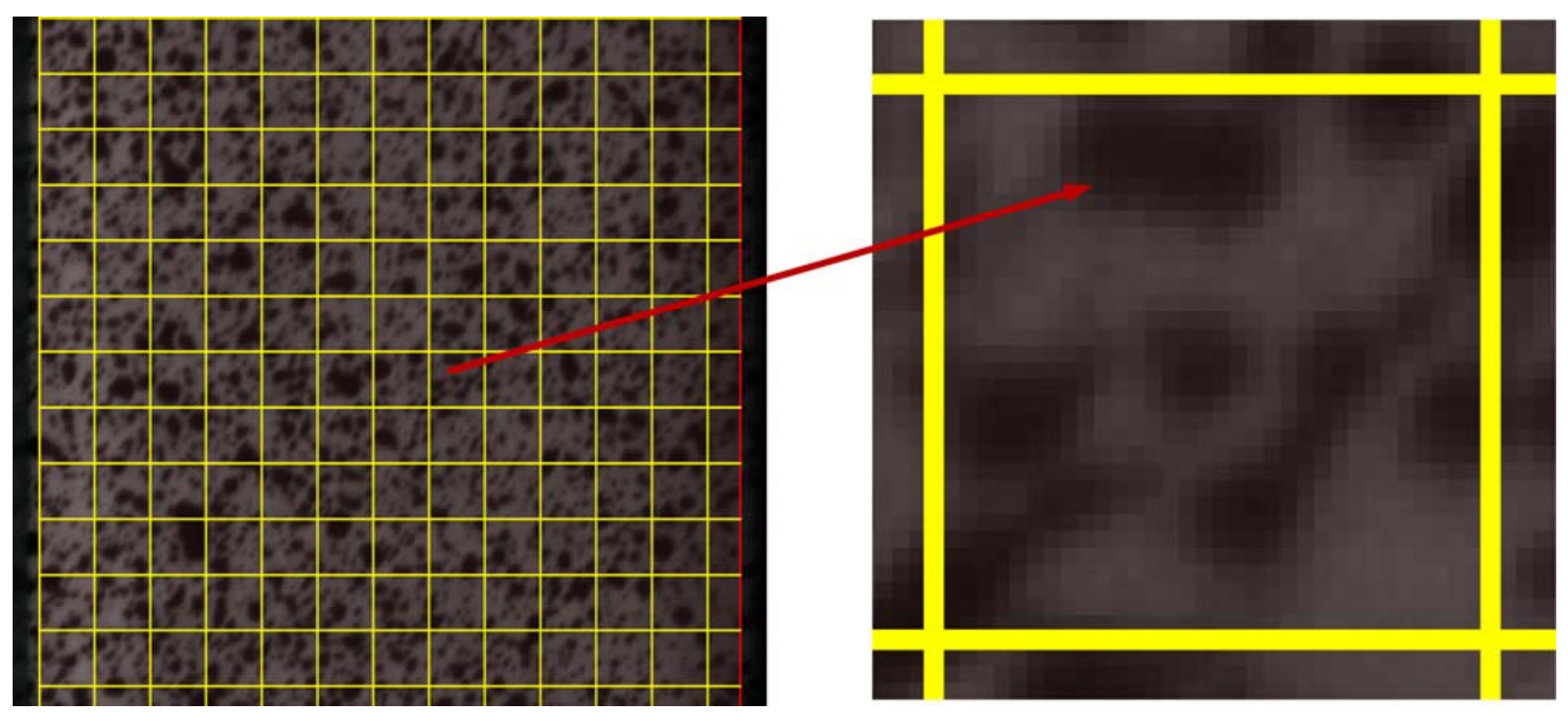

Fig. 5 Speckle image of the subsets with $33 \times 33$ pixels subset size.

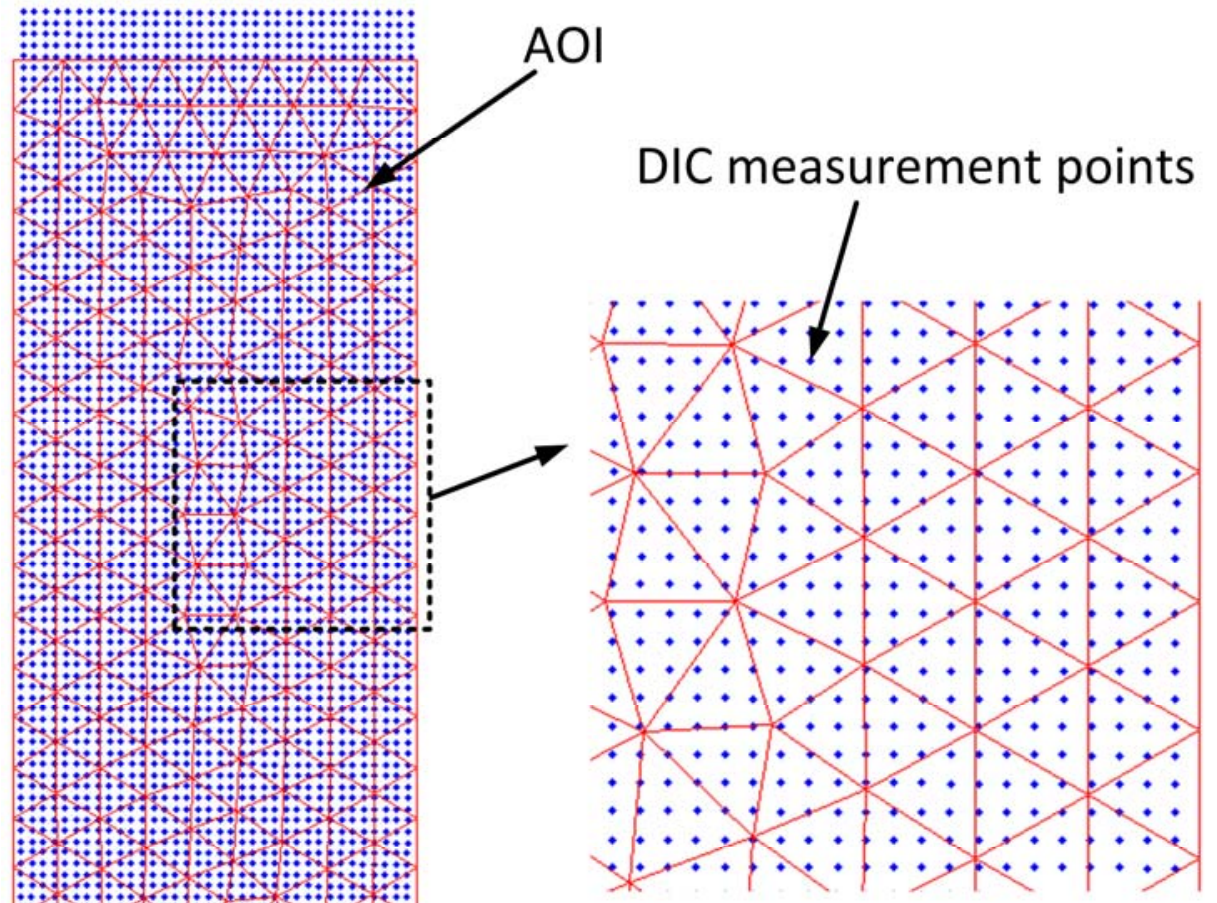

Fig. 6 Triangular mesh used for calculating the deformation gradient. 


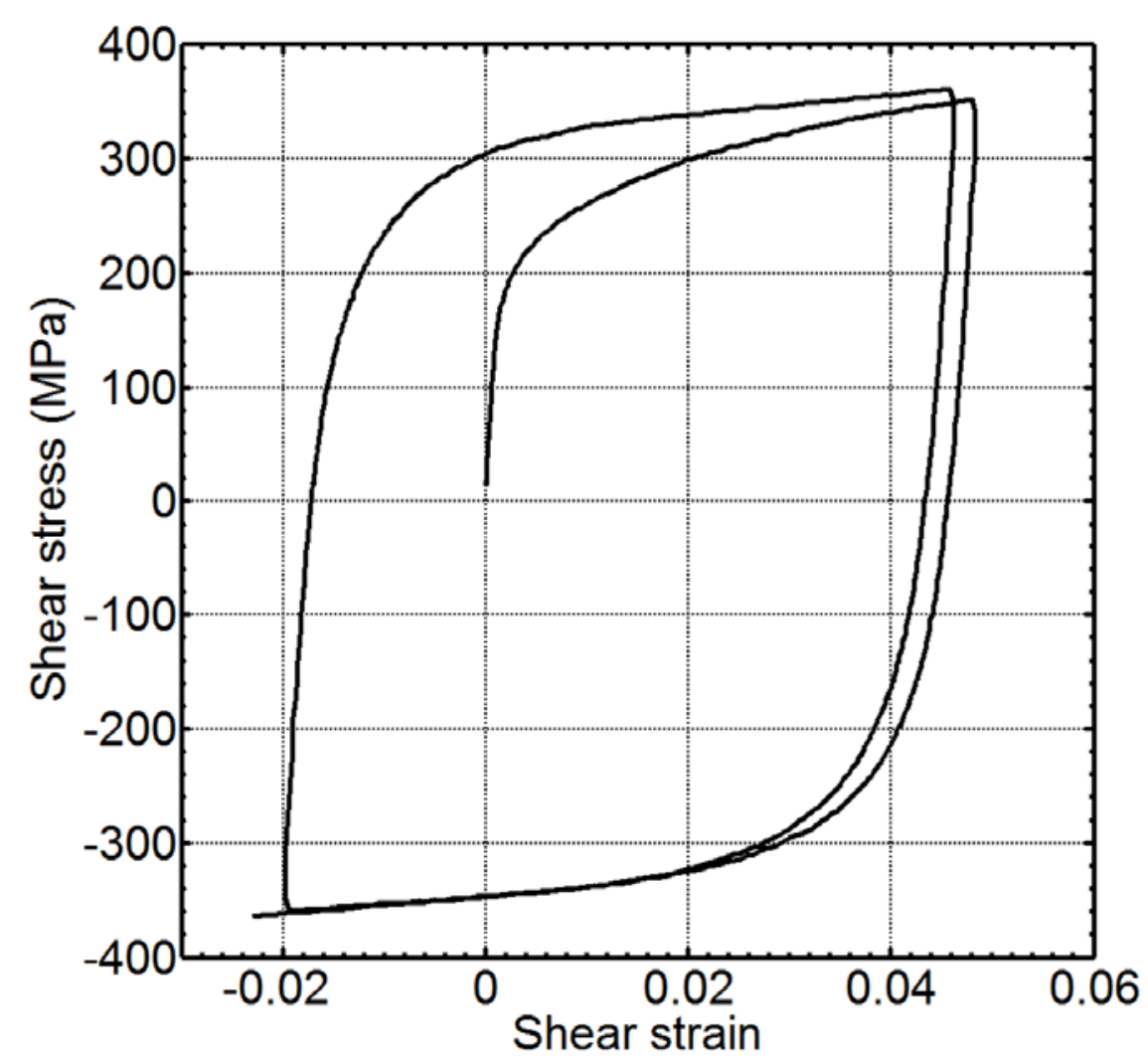

Fig. 7 Average shear stress-strain curve for the DP600 specimen 1 in two cycles of forwardreverse simple shear test

\subsection{Identification results for the AHSS}

The measured strain and load data were processed with the proposed VFM scheme. The virtual field VF1 in Eq. (19) was used for the identification. Since the identification results of the two specimens are very close to each other for each material, only one set of results will be presented here for each material for the sake of conciseness.

\subsubsection{DP600}

Two options are available when performing the identification. The HAH parameters and the Swift hardening parameters can be identified either simultaneously or separately. When identified separately, the Swift hardening parameters need to be identified first. This can be implemented using the isotropic hardening model (von Mises yield criterion for the present cases) for the first loading path of the tests as the HAH model can be reduced to isotropic hardening in the first loading path. In this case, the VFM program is adjusted for identifying only three unknown 
parameters $C, \varepsilon_{0}$ and $n$ of the Swift model. Two initial estimates were used to start the minimization. The identified Swift hardening parameters are given in Table 6. One can see that both initial estimates lead to values differing by about $1 \%$ at worst. Once the Swift hardening parameters are fixed (here, Identified 1 in Table 6 was used), the HAH parameters can be identified. Three different initial estimates were arbitrarily selected to cover the range of typical expected values. The identified HAH parameters are given in Table 7. It can be seen that for different initial estimates, the identified HAH parameters are in good agreement with each other. The difference in the cost function values that measure the gap between the IVW and EVW is also negligible. Fig. 8a shows the curves of the IVW and EVW when the minimization converges, which indicates that, overall, the two curves are in good agreement despite the slight deviations in parts of the curves, indicating that the identified results are reasonable.

On the other hand, the eight parameters (HAH and Swift hardening) can be identified simultaneously. As for the previous case, three initial estimates were attempted. The identification results are listed in Table 8. Again, the identified parameters are consistent for the different initial estimates. However, compared to the case of separated identification, different values were obtained, particularly for the Swift hardening parameters. Interestingly, the cost function values are lower for the simultaneous identification, which means that the gap between the IVW and EVW is decreased. This can be confirmed in Fig. 8b where the matching of the IVW and EVW curves is slightly improved compared to Fig. 8a. One explanation for this observation is that, for the former case, the Swift hardening parameters were identified based on only the first loading path, with $\sim 5 \%$ of total strain, while for the latter, the parameters were identified based on four loading paths, with over $20 \%$ of total strain. Hence, the parameters for the former case likely lead to be a local description of material mechanical behavior, while those for the latter can more comprehensively describe the material behavior. To understand this more clearly, future work needs to be done to determine the isotropic hardening parameters from a higher strain level using, e.g., bulge test and fixing these parameters to identify the HAH parameters, from which the influence of strain level on the identification results can be investigated. 
Table 6 Identified Swift hardening parameters for the DP600

\begin{tabular}{cccc}
\hline & $C$ & $\varepsilon_{0}$ & $n$ \\
\hline Initial estimate 1 & 2000 & 0.01 & 0.1 \\
Identified 1 & 1098 & 0.00343 & 0.199 \\
\hline Initial estimate 2 & 500 & 0.01 & 0.3 \\
\hline Identified 2 & 1100 & 0.00347 & 0.200 \\
\hline
\end{tabular}

Table 7 Separately identified HAH parameters for the DP600 (CFV: cost function value)

\begin{tabular}{|c|c|c|c|c|c|c|}
\hline & $k_{1}$ & $k_{2}$ & $k_{3}$ & $k_{4}$ & $k_{5}$ & $C F V$ \\
\hline Initial estimate 1 & 100 & 50 & 0.5 & 0.5 & 5 & \\
\hline Identified 1 & 128 & 66.8 & 0.35 & 0.56 & 10.1 & $7.80 \times 10^{7}$ \\
\hline Initial estimate 2 & 120 & 30 & 0.4 & 0.4 & 10 & \\
\hline Identified 2 & 130 & 64.1 & 0.32 & 0.56 & 10.3 & $7.72 \times 10^{7}$ \\
\hline Initial estimate 3 & 80 & 100 & 0.3 & 0.6 & 15 & \\
\hline Identified 3 & 130 & 62.8 & 0.32 & 0.57 & 10.6 & $7.73 \times 10^{7}$ \\
\hline
\end{tabular}

Table 8 Simultaneously identified HAH and Swift hardening parameters for the DP600

\begin{tabular}{|c|c|c|c|c|c|c|c|c|c|}
\hline & $k_{1}$ & $k_{2}$ & $k_{3}$ & $k_{4}$ & $k_{5}$ & $C$ & $\varepsilon_{0}$ & $n$ & $C F V$ \\
\hline Initial estimate 1 & 100 & 50 & 0.5 & 0.5 & 5 & 1200 & 0.01 & 0.2 & \\
\hline Identified 1 & 134 & 51.7 & 0.27 & 0.57 & 7.0 & 935 & $9.84 \times 10^{-4}$ & 0.151 & $6.14 \times 10^{7}$ \\
\hline Initial estimate 2 & 120 & 30 & 0.4 & 0.4 & 10 & 2000 & 0.01 & 0.1 & \\
\hline Identified 2 & 130 & 55.2 & 0.30 & 0.59 & 7.5 & 935 & $9.26 \times 10^{-4}$ & 0.151 & $6.12 \times 10^{7}$ \\
\hline Initial estimate 3 & 80 & 100 & 0.3 & 0.6 & 15 & 1000 & 0.005 & 0.15 & \\
\hline Identified 3 & 129 & 52.0 & 0.30 & 0.60 & 7.4 & 927 & $7.34 \times 10^{-4}$ & 0.147 & $6.17 \times 10^{7}$ \\
\hline
\end{tabular}




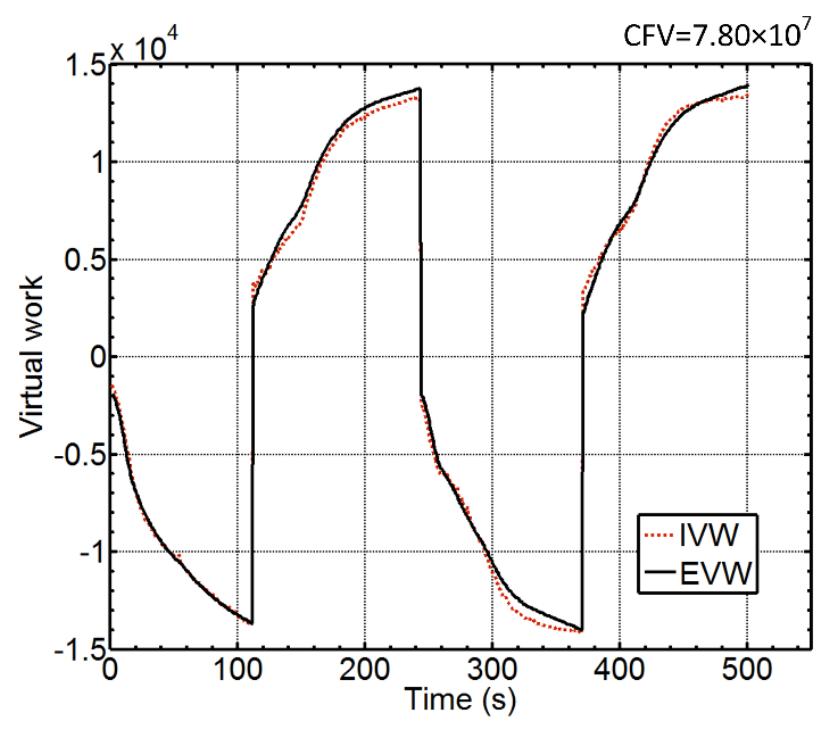

(a)

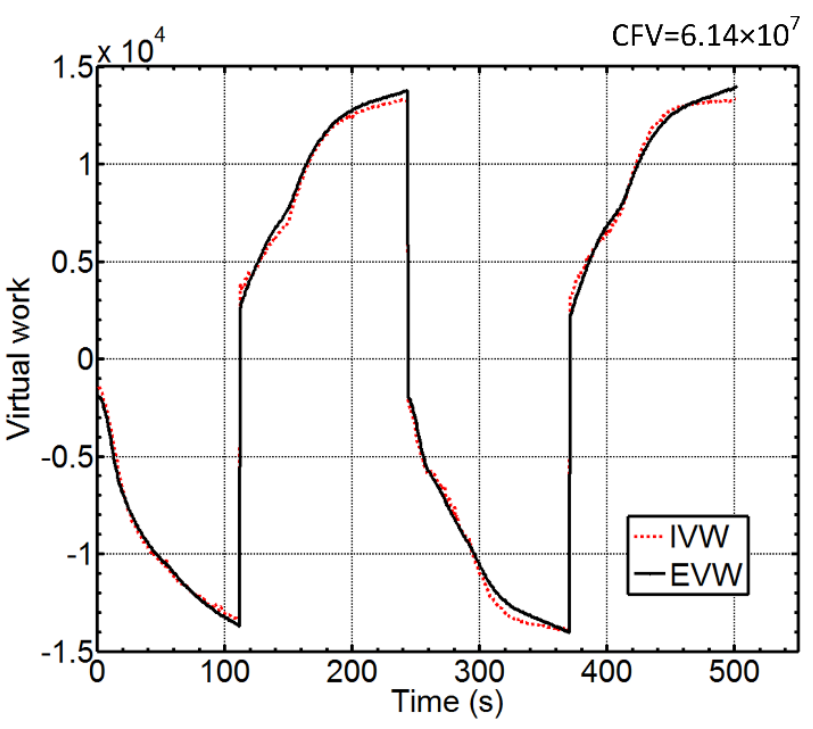

(b)

Fig. 8 Comparison of the internal and external virtual work for the DP600 specimen when the minimization converges (for initial estimate 1); (a) parameters identified separately (b) parameters identified simultaneously, CFV: cost function value.

The shear stress-strain curves calculated by the identified parameters are compared with the curves obtained from the experimental measurements in Fig. 9a. For the sake of legibility, these curves are also plotted with respect to the cumulated shear strain in Fig. 9b. The black dashed line was calculated from the parameters identified separately (Identified 1 in Table 6 and Table 7), while the green dash-dot line was calculated from the simultaneously identified parameters (Identified 1 in Table 8). Both of them are in good agreement with the experimental curve (red circles). It also can be observed that the green dash-dot curve matches the experimental curve slightly better. This confirms the results in Fig. 8, which show a smaller gap between the IVW and the EVW when the eight parameters are identified simultaneously. The curve calculated using the parameters of Identified 1 in Table 6 with the assumption of isotropic hardening is also plotted, from which one can clearly see the significance of the HAH model. Indeed, compared to the isotropic hardening, the material shows apparent Bauschinger effect and permanent softening after a load reversal. It should be mentioned that the shear stress-strain curves are shown here only for the parameters of Identified 1 since the difference is negligible when the curves are plotted for the other sets of results (Identified 2 and 3). Interestingly, in Fig. 9, one can see that the calculated curves obtained with the identified HAH parameters show a better match with the experimental curve in the forward loading paths (LP1 and LP3) than in the reverse loading paths (LP2 and LP4). 
This is due to the definition of the state variables $g_{3}$ and $g_{4}$ that control the material permanent softening. To adjust these discrepancies, the HAH model was modified, which is described in the discussion section.

Since the simultaneous identification provides a better solution over the separate identification, only the results of simultaneous identification will be given for the other materials in the following sections.

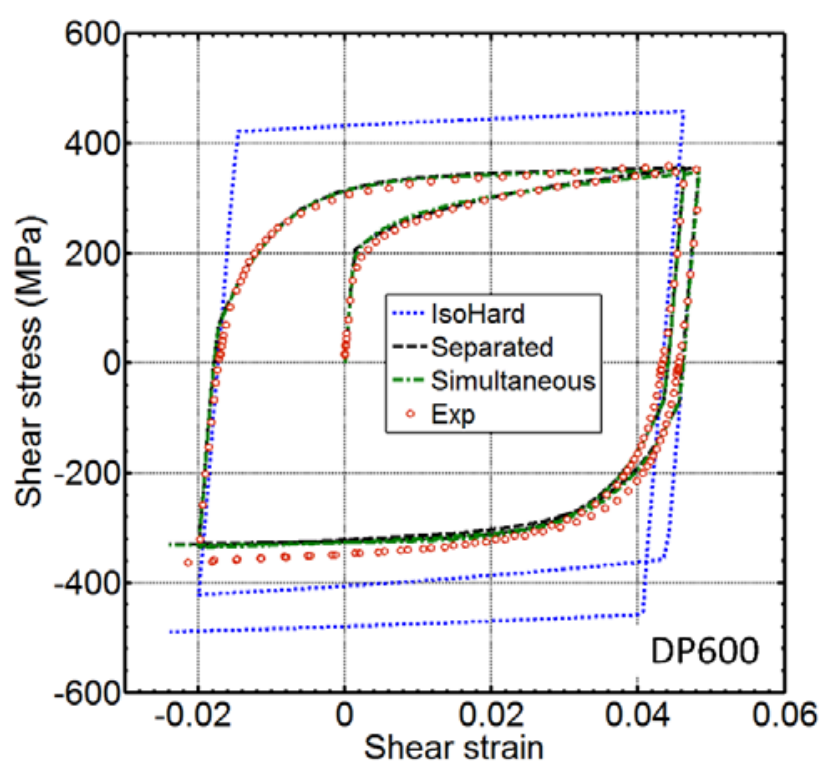

(a)

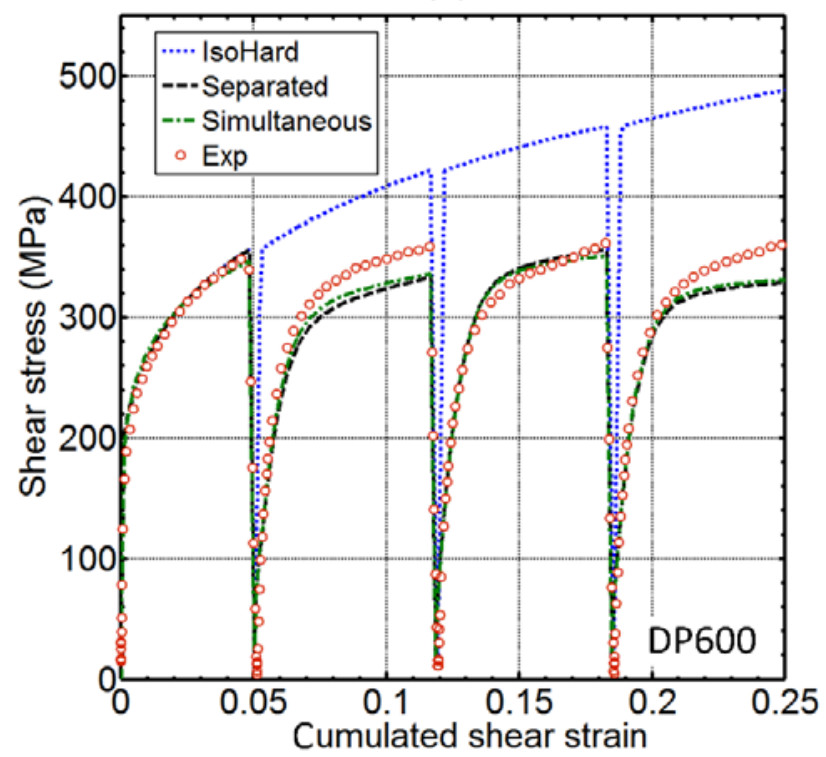

(b)

Fig. 9 Experimental, isotropic hardening and HAH calculated shear stress-strain curves for the DP600 specimen in two cycles of forward-reverse simple shear. (a) Plotted using true strain (b) Plotted using cumulated strain. 


\subsubsection{TRIP780 and TWIP980}

The identified HAH and Swift hardening parameters for the TRIP780 are listed in Table 9. Similar to the DP600, stable results were found for different initial estimates although an increase in the scatter of the identified $k_{4}$ and $k_{5}$, which are related to permanent softening, was observed. Fig. 10 illustrates the experimental, isotropic hardening and HAH calculated (using Identified 1) shear stress-strain curves. Again, the HAH calculated curve is in good agreement with the experimental counterpart. One can also see the considerable Bauschinger effect and permanent softening when comparing the HAH calculated curve to the isotropic hardening curve.

Finally, the identified parameters and the shear stress-strain curves for the TWIP980 are given in Table 10 and Fig. 11 (for Identified 1), from which the same conclusions as for the DP600 and TRIP780 can be drawn. For TWIP980 in Table 10, the deviations of the identified $\varepsilon_{0}$ are larger than those of the other parameters. This is not surprising because $\varepsilon_{0}$ is very sensitive to strain measurement and usually has larger identification error as can be seen in (Kim et al., 2014).

It is worth noting that similarly to the DP600, the experimental and HAH calculated stressstrain curves for both TRIP780 and TWIP980 are in better agreement in the forward loading paths than in the reverse loading paths, as can be observed in Fig. 10 and 11. A reason for this has been suggested in the previous section (due to the definition of the state variables $g_{3}$ and $g_{4}$ ). 
Table 9 Simultaneously identified HAH and Swift hardening parameters for the TRIP780

\begin{tabular}{|c|c|c|c|c|c|c|c|c|c|}
\hline & $k_{1}$ & $k_{2}$ & $k_{3}$ & $k_{4}$ & $k_{5}$ & C & $\varepsilon_{0}$ & $n$ & $C F V$ \\
\hline Initial estimate 1 & 100 & 50 & 0.5 & 0.5 & 5 & 1500 & 0.001 & 0.2 & \\
\hline Identified 1 & 121 & 137 & 0.28 & 0.51 & 2.6 & 1075 & $2.97 \times 10^{-3}$ & 0.145 & $1.34 \times 10^{8}$ \\
\hline Initial estimate 2 & 150 & 100 & 0.2 & 0.8 & 10 & 2000 & 0.01 & 0.1 & \\
\hline Identified 2 & 123 & 123 & 0.25 & 0.70 & 3.7 & 1045 & $2.57 \times 10^{-3}$ & 0.137 & $1.34 \times 10^{8}$ \\
\hline Initial estimate 3 & 50 & 200 & 0.4 & 0.8 & 15 & 500 & 0.01 & 0.1 & \\
\hline Identified 3 & 124 & 120 & 0.25 & 0.57 & 3.0 & 1084 & $3.56 \times 10^{-3}$ & 0.150 & $1.33 \times 10^{8}$ \\
\hline
\end{tabular}

Table 10 Simultaneously identified HAH and Swift hardening parameters for the TWIP980

\begin{tabular}{|c|c|c|c|c|c|c|c|c|c|}
\hline & $k_{1}$ & $k_{2}$ & $k_{3}$ & $k_{4}$ & $k_{5}$ & C & $\varepsilon_{0}$ & $n$ & $C F V$ \\
\hline Initial estimate 1 & 100 & 50 & 0.5 & 0.5 & 5 & 1500 & 0.001 & 0.1 & \\
\hline Identified 1 & 121 & 108 & 0.11 & 0.62 & 14.4 & 1321 & $1.99 \times 10^{-5}$ & 0.0849 & $1.60 \times 10^{8}$ \\
\hline Initial estimate 2 & 150 & 100 & 0.2 & 0.8 & 10 & 1000 & 0.001 & 0.1 & \\
\hline Identified 2 & 123 & 112 & 0.10 & 0.62 & 17.0 & 1401 & $3.48 \times 10^{-4}$ & 0.0996 & $1.64 \times 10^{8}$ \\
\hline Initial estimate 3 & 120 & 60 & 0.4 & 0.5 & 15 & 1200 & 0.001 & 0.15 & \\
\hline Identified 3 & 123 & 109 & 0.10 & 0.58 & 14.2 & 1374 & $6.88 \times 10^{-5}$ & 0.0942 & $1.60 \times 10^{8}$ \\
\hline
\end{tabular}




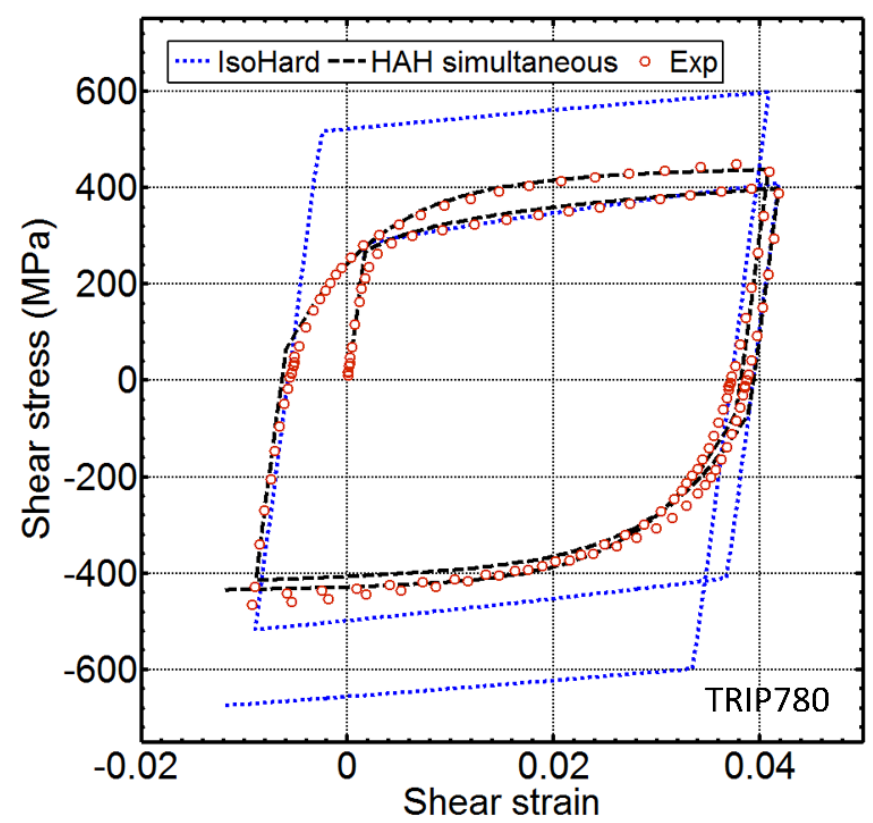

(a)

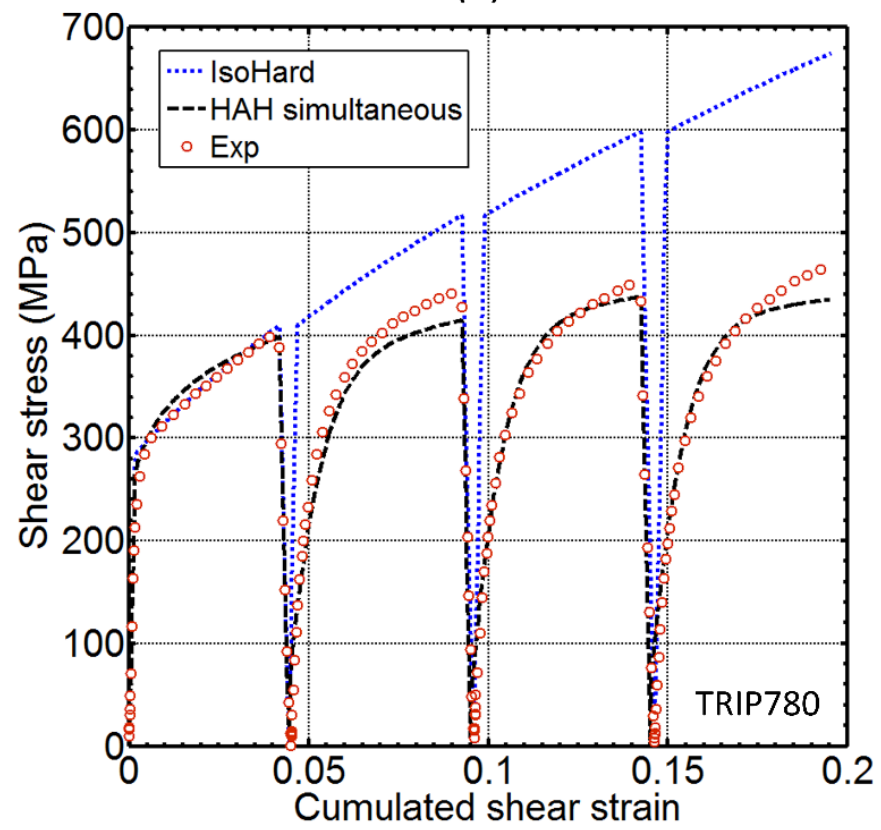

(b)

Fig. 10 Experimental, isotropic hardening and HAH calculated shear stress-strain curves for the TRIP780 specimen in two cycles of forward-reverse simple shear. (a) Plotted using true strain (b) Plotted using cumulated strain. 


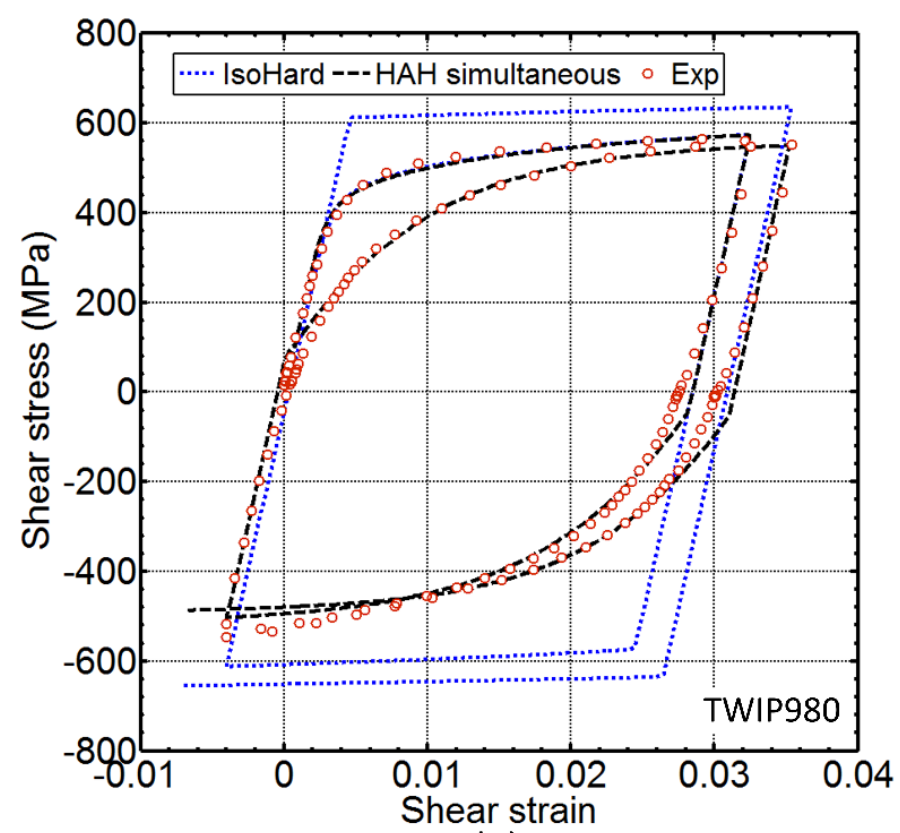

(a)

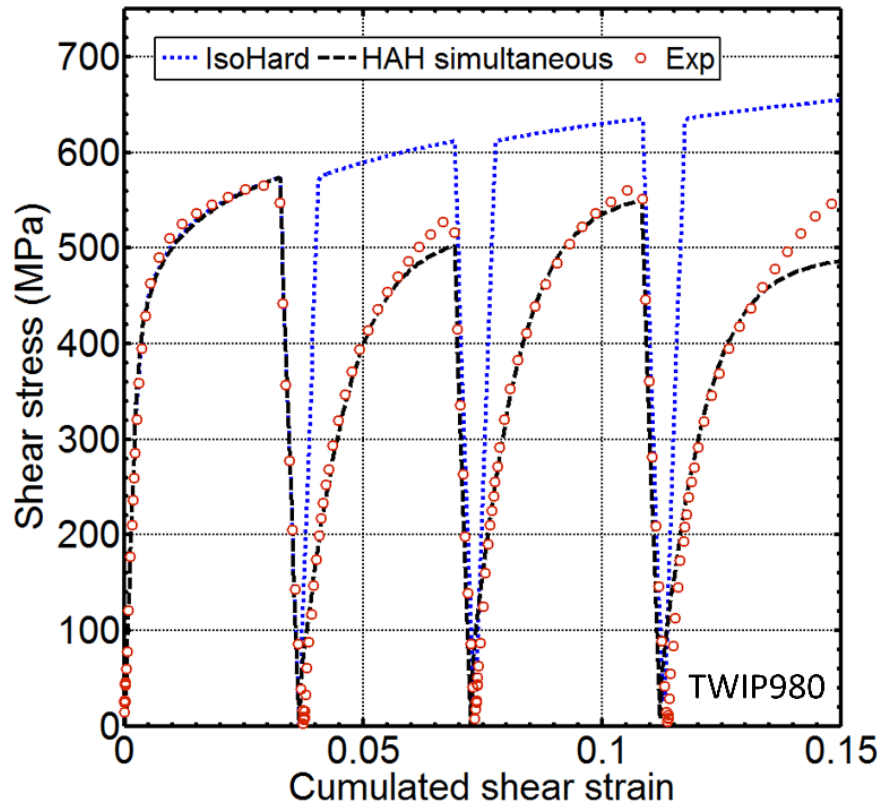

(b)

Fig. 11 Experimental, isotropic hardening and HAH calculated shear stress-strain curves for the TWIP980 specimen in two cycles of forward-reverse simple shear. (a) Plotted using true strain (b) Plotted using cumulated strain.

\section{Discussions}

As mentioned in the previous section, the discrepancies between the HAH calculated stressstrain curves and their experimental counterparts are higher in the reverse than in the forward 
loading paths. This indicates that the permanent softening is overestimated in the reverse loading paths. In order to understand these discrepancies more clearly, the evolutions of the permanent softening related state variables $g_{3}$ and $g_{4}$ are plotted in blue dot and dashed lines, respectively, in Fig. 12 for the DP600. In Eqs. (5) and (6), the state variables that are used to account for the permanent softening are $g_{3}$ and $g_{4}$. In LP2, for which $\widehat{\boldsymbol{h}}: \boldsymbol{s}<0$ in Eq. (6), $g_{4}$ is involved in the evolution of the state variable $g_{l}$, and thus determines permanent softening, whereas $g_{3}$ in this loading step only evolves but is not implicated in the determination of permanent softening. In LP3, the roles of $g_{3}$ and $g_{4}$ are switched and their evolutions follow the rules in Eq. (5). In this case $\left(\widehat{\boldsymbol{h}}: \boldsymbol{s} \geq 0\right.$ ), $g_{3}$ determines permanent softening by its involvement in the evolution of the state variable $g_{2}$, whereas $g_{4}$ in this loading step only evolves but is not used. The same analysis also applies to the subsequent loading steps. Therefore, in LP2, LP3, and LP4, the state variables that are used to account for permanent softening are $g_{4}, g_{3}$ and $g_{4}$, respectively, and are referred to as $g_{40}$ used, $g_{30}$ used and $g_{40}$ used (o is the abbreviation for original HAH model), respectively, in Fig. 12. The other state variables in LP2, LP3, and LP4, namely $g_{3}, g_{4}$, and $g_{3}$, are not used but evolve, which is designated as $g_{30}$ evolves, $g_{40}$ evolves and $g_{30}$ evolves, respectively, in Fig. 12. One can clearly see that, for LP2 and LP4, there are much more significant decreases in the used state variables, namely D2 and D4, while for LP1 and LP3, the decreases D1 and D3 are much smaller (D1 equals zero since there is no permanent softening in monotonic loading). This is consistent with the larger permanent softening observed in the HAH calculated stress-strain curves for LP2 and LP4 in Fig. 9-11. However, by inspection of the corresponding experimental stressstrain curves, it appears that the permanent softening is continuous in all loading paths. Therefore, in order to adjust these discrepancies, the original HAH model has been modified. The idea is to define a continuous permanent softening to adapt to the experimental results but also maintain the advantage of the original HAH model, in which the yield surface, in the case of monotonic loading, remains identical to that of isotropic hardening on the side of the active stress. Based on this idea, in the modified HAH model, permanent softening is a function of $g_{3}$ only instead of using two state variables $g_{3}$ and $g_{4}$. A new parameter $P_{0}$ is introduced. As long as $\widehat{\boldsymbol{h}}: \boldsymbol{s}$ is positive (monotonic or pseudo-monotonic loading), $P_{0}$ equals zero. As soon as $\widehat{\boldsymbol{h}}$ : $\boldsymbol{s}$ changes sign, $P_{0}$ takes value of one and remains equal to one, allowing the activation of permanent softening. Thus,

$$
\begin{gathered}
P_{0}=0, \text { in monotonic loading } \\
P_{0}=1, \text { after } \widehat{\boldsymbol{h}}: \boldsymbol{s} \text { changes sign }
\end{gathered}
$$


and the evolution of $g_{3}$ writes

$$
\frac{d g_{3}}{d \bar{\varepsilon}}=P_{0} k_{5}\left(k_{4}-g_{3}\right)
$$

This means that the permanent softening becomes effective only after the first load reversal or pseudo-reversal. The evolutions of the other state variables become:

For $\widehat{\boldsymbol{h}}: \boldsymbol{s} \geq 0$

$$
\begin{aligned}
& \frac{d g_{1}}{d \bar{\varepsilon}}=k_{2}\left(k_{3} \frac{\sigma(0)}{\sigma(\bar{\varepsilon})}-g_{1}\right) \\
& \frac{d g_{2}}{d \bar{\varepsilon}}=k_{1} \frac{g_{3}-g_{2}}{g_{2}} \\
& \frac{d \widehat{\boldsymbol{h}}}{d \bar{\varepsilon}}=k\left[\hat{\boldsymbol{s}}-\frac{8}{3} \widehat{\boldsymbol{h}}(\hat{\boldsymbol{s}}: \widehat{\boldsymbol{h}})\right]
\end{aligned}
$$

For $\widehat{\boldsymbol{h}}: \boldsymbol{s}<0$

$$
\begin{aligned}
& \frac{d g_{1}}{d \bar{\varepsilon}}=k_{1} \frac{g_{3}-g_{1}}{g_{1}} \\
& \frac{d g_{2}}{d \bar{\varepsilon}}=k_{2}\left(k_{3} \frac{\sigma(0)}{\sigma(\bar{\varepsilon})}-g_{2}\right) \\
& \frac{d \widehat{\boldsymbol{h}}}{d \bar{\varepsilon}}=k\left[-\widehat{\boldsymbol{s}}+\frac{8}{3} \widehat{\boldsymbol{h}}(\hat{\boldsymbol{s}}: \widehat{\boldsymbol{h}})\right]
\end{aligned}
$$

The proposed VFM scheme has been updated based on the new HAH formulation and applied to the experimental results. The simultaneously identified HAH and Swift parameters are listed in Table 11-13 for the DP600, TRIP780 and TWIP980, respectively. It is worth noting that the identified $k_{4}$ and $k_{5}$ are more stable now for the TRIP780 regardless of different initial estimates compared to the results in Table 9. Using the identified parameters (Identified 1) of the DP600, the evolution of the state variable $g_{3}$ for the modified HAH model was calculated and plotted in red solid curve in Fig. 12. It is clear that the evolution of $g_{3}$ now is continuous. The shear stress-strain curves calculated by the modified HAH model using the identified parameters (Identified 1) in Table 11-13 are illustrated in Fig. 13 for the tested materials. The new version of the model significantly reduces that discrepancies observed with the old version for LP2 and LP4. It should be pointed out that this modification might not be the optimal, and further improvement still needs to be attempted to provide a better prediction of the material behavior. Nevertheless, at this stage, this modification is satisfactory. 


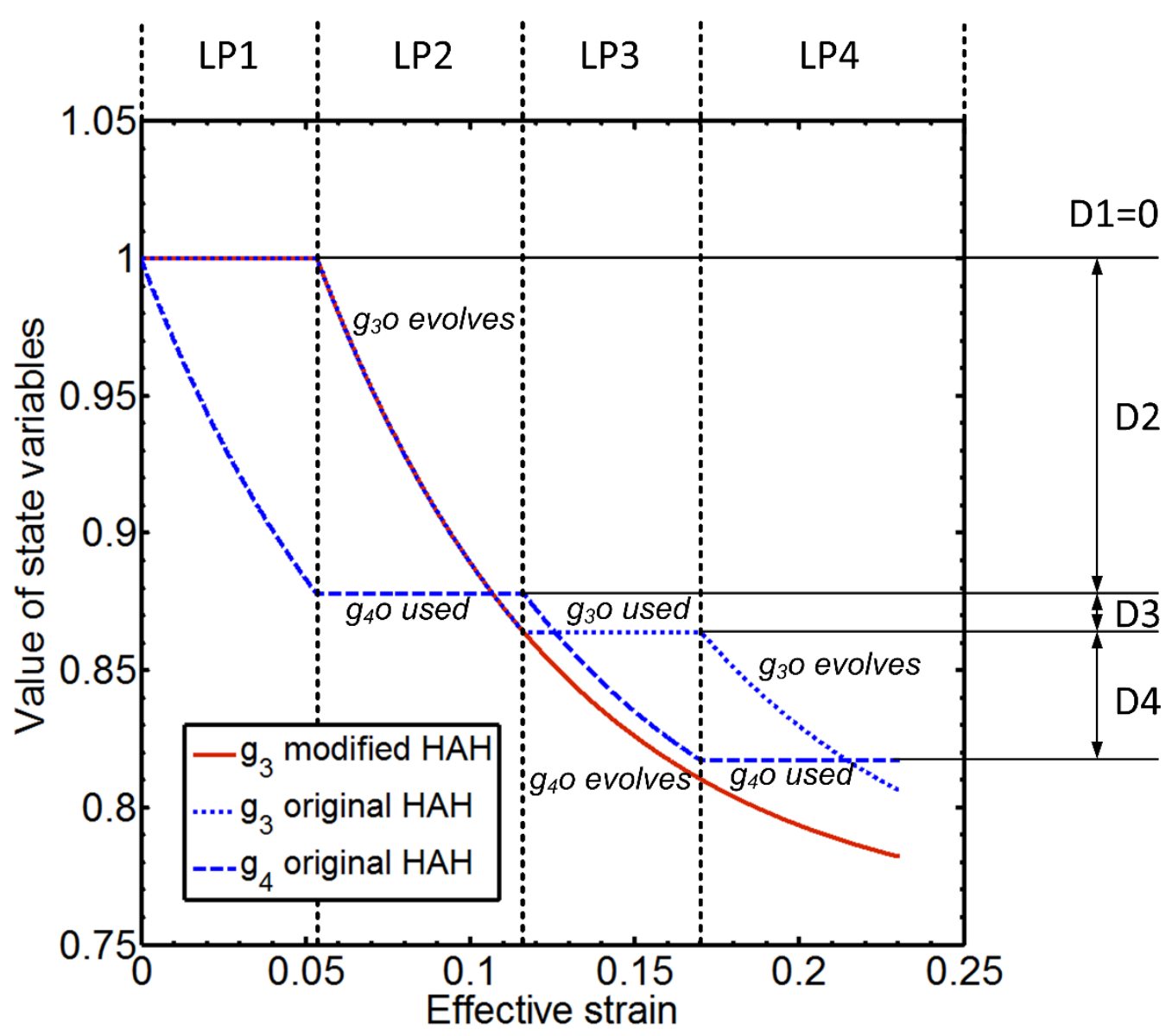

Fig. 12 Evolutions of the permanent softening related state variables for the DP600 obtained with the original and modified HAH models

Table 11 Simultaneously identified HAH and Swift hardening parameters for the DP600 obtained with the modified HAH model

\begin{tabular}{|c|c|c|c|c|c|c|c|c|c|}
\hline & $k_{1}$ & $k_{2}$ & $k_{3}$ & $k_{4}$ & $k_{5}$ & $C$ & $\varepsilon_{0}$ & $n$ & $C F V$ \\
\hline Initial estimate 1 & 100 & 50 & 0.5 & 0.5 & 5 & 1200 & 0.01 & 0.2 & \\
\hline Identified 1 & 110 & 128 & 0.42 & 0.76 & 13.2 & 989 & $2.25 \times 10^{-3}$ & 0.171 & $6.60 \times 10^{7}$ \\
\hline Initial estimate 2 & 120 & 30 & 0.4 & 0.4 & 10 & 2000 & 0.01 & 0.1 & \\
\hline Identified 2 & 117 & 125 & 0.37 & 0.78 & 12.6 & 935 & $1.74 \times 10^{-3}$ & 0.155 & $6.57 \times 10^{7}$ \\
\hline Initial estimate 3 & 80 & 100 & 0.3 & 0.6 & 15 & 1000 & 0.005 & 0.15 & \\
\hline Identified 3 & 111 & 117 & 0.40 & 0.77 & 13.7 & 966 & $2.14 \times 10^{-3}$ & 0.164 & $6.55 \times 10^{7}$ \\
\hline
\end{tabular}


Table 12 Simultaneously identified HAH and Swift hardening parameters for the TRIP780 obtained with the modified HAH model

\begin{tabular}{|c|c|c|c|c|c|c|c|c|c|}
\hline & $k_{1}$ & $k_{2}$ & $k_{3}$ & $k_{4}$ & $k_{5}$ & $C$ & $\varepsilon_{0}$ & $n$ & $C F V$ \\
\hline Initial estimate 1 & 100 & 50 & 0.5 & 0.5 & 5 & 1500 & 0.001 & 0.2 & \\
\hline Identified 1 & 115 & 157 & 0.32 & 0.77 & 6.2 & 1246 & $7.49 \times 10^{-3}$ & 0.197 & $1.04 \times 10^{8}$ \\
\hline Initial estimate 2 & 150 & 100 & 0.2 & 0.8 & 10 & 2000 & 0.01 & 0.1 & \\
\hline Identified 2 & 119 & 159 & 0.30 & 0.78 & 7.7 & 1271 & $8.53 \times 10^{-3}$ & 0.206 & $1.04 \times 10^{8}$ \\
\hline Initial estimate 3 & 50 & 200 & 0.4 & 0.8 & 15 & 500 & 0.01 & 0.1 & \\
\hline Identified 3 & 120 & 170 & 0.30 & 0.78 & 7.7 & 1282 & $9.07 \times 10^{-3}$ & 0.209 & $1.03 \times 10^{8}$ \\
\hline
\end{tabular}

Table 13 Simultaneously identified HAH and Swift hardening parameters for the TWIP980 obtained with the modified HAH model

\begin{tabular}{|c|c|c|c|c|c|c|c|c|c|}
\hline & $k_{1}$ & $k_{2}$ & $k_{3}$ & $k_{4}$ & $k_{5}$ & C & $\varepsilon_{0}$ & $n$ & CFV \\
\hline Initial estimate 1 & 100 & 50 & 0.5 & 0.5 & 5 & 1500 & 0.001 & 0.1 & \\
\hline Identified 1 & 84.1 & 71.6 & 0.10 & 0.65 & 3.5 & 1249 & $7.02 \times 10^{-6}$ & 0.0739 & $3.50 \times 10^{8}$ \\
\hline Initial estimate 2 & 150 & 100 & 0.2 & 0.8 & 10 & 1000 & 0.001 & 0.1 & \\
\hline Identified 2 & 88.8 & 75.5 & 0.11 & 0.70 & 3.7 & 1221 & $1.01 \times 10^{-6}$ & 0.0706 & $3.62 \times 10^{8}$ \\
\hline Initial estimate 3 & 120 & 60 & 0.4 & 0.5 & 15 & 1200 & 0.001 & 0.15 & \\
\hline Identified 3 & 85.2 & 72.8 & 0.10 & 0.65 & 3.5 & 1259 & $2.88 \times 10^{-5}$ & 0.0777 & $3.60 \times 10^{8}$ \\
\hline
\end{tabular}




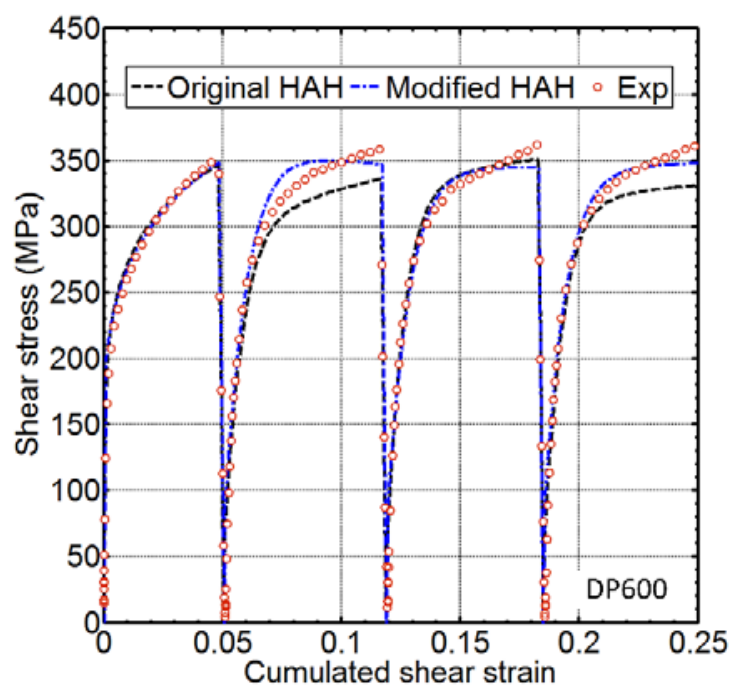

(a)

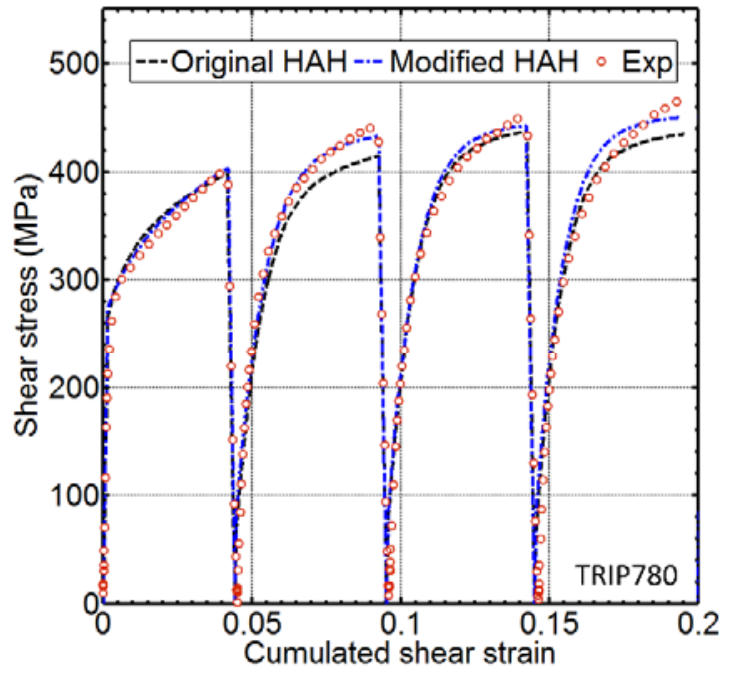

(b)

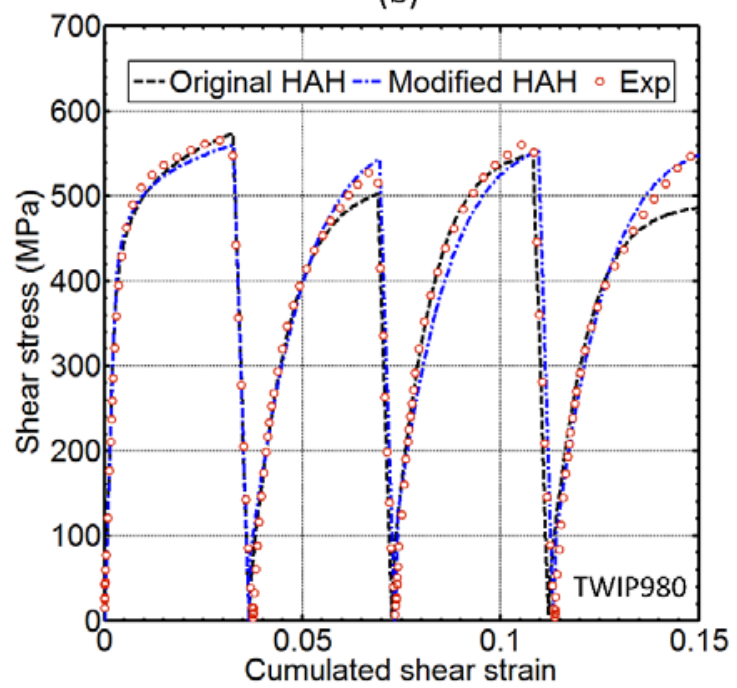

(c)

Fig. 13 Experimental and HAH (the original and modified) calculated shear stress-strain curves for: (a) DP600 (b) TRIP780 (c) TWIP980 


\section{Conclusions}

In this paper, the virtual field method (VFM) was extended to the HAH model in order to characterize the material mechanical behavior when subjected to a few load cycles. Specifically, the objective was to use the VFM to identify, from simple shear cycle tests, the HAH constitutive parameters that determine the Bauschinger effect and permanent softening in metal forming. The identification method is based on developing a cost function that measures the gap between the total internal and external virtual works of the whole deformation process and minimizing it with respect to the unknown constitutive parameters. The main conclusions of the present work are as follows.

- The proposed identification method was validated on a FE model of forward-reverse simple shear, in which the input constitutive parameters of the FE model were successfully retrieved using the proposed VFM scheme. It was found that at least two forward-reverse loading cycles are needed to provide an accurate identification. A random Gaussian white noise was added to the simulation results, from which the influence of measurement noise on the identification results was evaluated.

- Reasonable constitutive parameters were obtained for the selected AHSS, namely DP600, TRIP780 and TWIP980, using the proposed identification method. Good agreement was found between the HAH calculated shear stress-strain curves and their experimental counterparts despite of the larger discrepancies observed in the reverse loading paths compared to forward loading paths. These discrepancies were attributed to the definition of the state variables that control permanent softening in the HAH model.

- The original HAH model was modified to provide a continuous evolution for the permanent softening related state variables in order to adjust the observed discrepancies. In the modified model, there is only one state variable controlling the permanent softening.

- The VFM scheme was updated for the modified HAH formulation and then applied to the experimental data. The shear stress-strain curves were well described using the identified constitutive parameters obtained with the modified HAH model. It was found that after modification the discrepancies observed in the reverse loading paths for the original HAH model were significantly reduced. 


\section{Acknowledgments}

The authors (Dr. Jiawei Fu, Prof. Frédéric Barlat and Dr. Jin-Hwan Kim) are grateful to POSCO for financial support. Prof. F. Pierron is thankful to the Royal Society and the Wolfson Foundation for providing him support through a Royal Society Wolfson Research Merit Award.

\section{References}

Avril, S., Badel, P., Duprey, A., 2010. Anisotropic and hyperelastic identification of in vitro human arteries from full-field optical measurements. Journal of biomechanics 43, 2978-2985.

Avril, S., Bonnet, M., Bretelle, A.S., Grédiac, M., Hild, F., Ienny, P., Latourte, F., Lemosse, D., Pagano, S., Pagnacco, E., Pierron, F., 2008. Overview of identification methods of mechanical parameters based on full-field measurements. Experimental Mechanics 48, 381-402.

Barlat, F., Brem, J.C., Yoon, J.W., Chung, K., Dick, R.E., Lege, D.J., Pourboghrat, F., Choi, S.H., Chu, E., 2003. Plane stress yield function for aluminum alloy sheets_part 1: theory. International Journal of Plasticity 19, 1297-1319.

Barlat, F., Gracio, J.J., Lee, M.-G., Rauch, E.F., Vincze, G., 2011. An alternative to kinematic hardening in classical plasticity. International Journal of Plasticity 27, 1309-1327.

Barlat, F., Ha, J., Grácio, J.J., Lee, M.-G., Rauch, E.F., Vincze, G., 2013. Extension of homogeneous anisotropic hardening model to cross-loading with latent effects. International Journal of Plasticity 46, 130-142.

Barlat, F., Vincze, G., Grácio, J.J., Lee, M.G., Rauch, E.F., Tomé, C.N., 2014. Enhancements of homogenous anisotropic hardening model and application to mild and dual-phase steels. International Journal of Plasticity 58, 201-218.

Broggiato, G., Campana, F., Cortese, L., 2008. The Chaboche nonlinear kinematic hardening model: calibration methodology and validation. Meccanica 43, 115-124.

Chaboche, J.L., 1986. Time-independent constitutive theories for cyclic plasticity. International Journal of Plasticity 2, 149-188.

Chaboche, J.L., 2008. A review of some plasticity and viscoplasticity constitutive theories. International Journal of Plasticity 24, 1642-1693. 
Choi, J.S., Lee, J.W., Kim, J.H., Barlat, F., Lee, M.G., Kim, D., 2015. Measurement and modeling of simple shear deformation under load reversal: Application to advanced high strength steels. International Journal of Mechanical Sciences 98, 144-156.

Chung, K., Lee, M.-G., Kim, D., Kim, C., Wenner, M.L., Barlat, F., 2005. Spring-back evaluation of automotive sheets based on isotropic-kinematic hardening laws and non-quadratic anisotropic yield functions: Part I: theory and formulation. International Journal of Plasticity 21, 861-882.

Chung, K., Ma, N., Park, T., Kim, D., Yoo, D., Kim, C., 2011. A modified damage model for advanced high strength steel sheets. International Journal of Plasticity 27, 1485-1511.

Dafalias, Y.F., Popov, E.P., 1975. A model of nonlinearly hardening materials for complex loading. Acta Mechanica 21, 173-192.

Firat, M., Kaftanoglu, B., Eser, O., 2008. Sheet metal forming analyses with an emphasis on the springback deformation. Journal of Materials Processing Technology 196, 135-148.

Frederick, C.O., Armstrong, P.J., 2007. A mathematical representation of the multiaxial Bauschinger effect. Materials at High Temperatures 24, 1-26.

Fu, J., Pierron, F., Ruiz, P.D., 2013. Elastic stiffness characterization using three-dimensional fullfield deformation obtained with optical coherence tomography and digital volume correlation. J Biomed Opt 18, 121512.

Geng, L., Wagoner, R.H., 2002. Role of plastic anisotropy and its evolution on springback. International Journal of Mechanical Sciences 44, 123-148.

Grama, S.N., Subramanian, S.J., Pierron, F., 2015. On the identifiability of Anand visco-plastic model parameters using the Virtual Fields Method. Acta Materialia 86, 118-136.

Grédiac, M., Pierron, F., 2006. Applying the Virtual Fields Method to the identification of elastoplastic constitutive parameters. International Journal of Plasticity 22, 602-627.

Grédiac, M., Sur, F., Blaysat, B., 2016. The grid method for in-plane displacement and strain measurement: a review and analysis. Strain, In press.

Güner, A., Soyarslan, C., Brosius, A., Tekkaya, A.E., 2012. Characterization of anisotropy of sheet metals employing inhomogeneous strain fields for Yld2000-2D yield function. International Journal of Solids and Structures 49, 3517-3527.

Haddag, B., Balan, T., Abed-Meraim, F., 2007. Investigation of advanced strain-path dependent material models for sheet metal forming simulations. International Journal of Plasticity 23, 951979. 
Kim, J.H., Avril, S., Duprey, A., Favre, J.P., 2012. Experimental characterization of rupture in human aortic aneurysms using a full-field measurement technique. Biomechanics and modeling in mechanobiology 11, 841-853.

Kim, J.H., Barlat, F., Pierron, F., Lee, M.G., 2014. Determination of Anisotropic Plastic Constitutive Parameters Using the Virtual Fields Method. Experimental Mechanics 54, 11891204.

Kim, J.H., Serpantié, A., Barlat, F., Pierron, F., Lee, M.G., 2013. Characterization of the postnecking strain hardening behavior using the virtual fields method. International Journal of Solids and Structures 50, 3829-3842.

Lecompte, D., Smits, A., Sol, H., Vantomme, J., Van Hemelrijck, D., 2007. Mixed numericalexperimental technique for orthotropic parameter identification using biaxial tensile tests on cruciform specimens. International Journal of Solids and Structures 44, 1643-1656.

Lee, J.-W., Lee, M.-G., Barlat, F., 2012. Finite element modeling using homogeneous anisotropic hardening and application to spring-back prediction. International Journal of Plasticity 29, 13-41. Lee, M.-G., Kim, D., Kim, C., Wenner, M.L., Chung, K., 2005a. Spring-back evaluation of automotive sheets based on isotropic-kinematic hardening laws and non-quadratic anisotropic yield functions, part III: applications. International Journal of Plasticity 21, 915-953.

Lee, M.G., Kim, D., Kim, C., Wenner, M.L., Chung, K., 2005b. Spring-back evaluation of automotive sheets based on isotropic-kinematic hardening laws and non-quadratic anisotropic yield functions, part III: Applications. International Journal of Plasticity 21, 915-953.

Ohno, N., Wang, J.D., 1993. Kinematic hardening rules with critical state of dynamic recovery, part I: formulation and basic features for ratchetting behavior. International Journal of Plasticity 9 , 375-390.

Pierron, F., Avril, S., Tran, V.T., 2010. Extension of the virtual fields method to elasto-plastic material identification with cyclic loads and kinematic hardening. International Journal of Solids and Structures 47, 2993-3010.

Pierron, F., Grediac, M., 2012. The virtual fields method: Extracting constitutive mechanical parameters from full-field deformation measurements.

Prager, W., 1949. Recent Developments in the Mathematical Theory of Plasticity. Journal of Applied Physics 20, 235-241. 
Rossi, M., Lava, P., Pierron, F., Debruyne, D., Sasso, M., 2015. Effect of DIC Spatial Resolution, Noise and Interpolation Error on Identification Results with the VFM. Strain 51, 206-222.

Sutton, M.A., Deng, X., Liu, J., Yang, L., 1996. Determination of elastic-plastic stresses and strains from measured surface strain data. Experimental Mechanics 36, 99-112.

Sutton, M.A., Orteu, J.-J., Schreier, H., 2009. Image Correlation for Shape, Motion and Deformation Measurements: Basic Concepts,Theory and Applications. Springer Publishing Company, Incorporated.

Wang, P., Pierron, F., Rossi, M., Lava, P., Thomsen, O.T., 2016. Optimised Experimental Characterisation of Polymeric Foam Material Using DIC and the Virtual Fields Method. Strain 52, 59-79.

Wang, W., Mottershead, J.E., Sebastian, C.M., Patterson, E.A., 2011. Shape features and finite element model updating from full-field strain data. International Journal of Solids and Structures 48, 1644-1657.

Yanagimoto, J., Oyamada, K., Nakagawa, T., 2005. Springback of High-Strength Steel after Hot and Warm Sheet Formings. CIRP Annals - Manufacturing Technology 54, 213-216.

Yoshida, F., Hamasaki, H., Uemori, T., 2015. Modeling of anisotropic hardening of sheet metals including description of the Bauschinger effect. International Journal of Plasticity 75, 170-188.

Yoshida, F., Uemori, T., 2003. A model of large-strain cyclic plasticity and its application to springback simulation. International Journal of Mechanical Sciences 45, 1687-1702.

Zang, S.-1., Lee, M.-G., Sun, L., Kim, J.H., 2014. Measurement of the Bauschinger behavior of sheet metals by three-point bending springback test with pre-strained strips. International Journal of Plasticity 59, 84-107.

Zhang, S., Leotoing, L., Guines, D., Thuillier, S., Zang, S.-1., 2014. Calibration of anisotropic yield criterion with conventional tests or biaxial test. International Journal of Mechanical Sciences 85, 142-151.

Ziegler, H., 1959. A modification of Prager's hardening rule. Quarterly of Applied Mathematics. 